\title{
ESTIMATES OF THE FIRST DIRICHLET EIGENVALUE FROM EXIT TIME MOMENT SPECTRA
}

\author{
A. HURTADO ${ }^{\natural}$, S. MARKVORSEN" ${ }^{\#}$, AND V. PALMER*
}

\begin{abstract}
We compute the first Dirichlet eigenvalue of a geodesic ball in a rotationally symmetric model space in terms of the moment spectrum for the Brownian motion exit times from the ball. As an application of the model space theory we prove lower and upper bounds for the first Dirichlet eigenvalues of extrinsic metric balls in submanifolds of ambient Riemannian spaces which have model space controlled curvatures. Moreover, from this general setting we thereby obtain new generalizations of the classical and celebrated results due to McKean and Cheung-Leung concerning the fundamental tones of Cartan-Hadamard manifolds and the fundamental tones of submanifolds with bounded mean curvature in hyperbolic spaces, respectively.
\end{abstract}

\section{INTRODUCTION}

Given a complete Riemannian manifold $\left(N^{n}, g\right)$ and a normal domain $D \subset N$, we denote by $\lambda_{1}(D)$ the first eigenvalue of the Dirichlet boundary value problem

$$
\begin{aligned}
\Delta u+\lambda u & =0 \text { on } D \\
\left.u\right|_{\partial D} & =0,
\end{aligned}
$$

where $\Delta$ denotes the Laplace-Beltrami operator on $\left(N^{n}, g\right)$. The increasing sequence of eigenvalues $\left\{\lambda_{k}(D)\right\}_{k=1}^{\infty}$ for this problem is the Dirichlet spectrum of $D$.

When we consider $D=B^{N}(p, r)$, the geodesic $r$-balls centered at a given point $p \in N$, the first eigenvalue $\lambda_{1}(r)=\lambda_{1}\left(B^{N}(p, r)\right)$ is a decreasing function of $r$ and the limit at $r \rightarrow \infty$ does not depend on the choice of center for the balls $p$. This is a consequence of the Domain Monotonicity Principle. The exhaustion of $N$ by geodesic balls $\left\{B^{N}(p, r)\right\}_{r \rightarrow \infty}$ thence produces the Dirichlet fundamental tone of the manifold N:

$$
\lambda^{*}(N)=\lim _{r \rightarrow \infty} \lambda_{1}\left(B^{N}(p, r)\right)
$$

The calculation of the exact values of the first eigenvalue $\lambda_{1}(D)$ of a (small or large) normal domain $D \subset N$ in a given complete Riemannian manifold $N$ by establishing its relationship with different geometric invariants is an open question, even when we consider

2000 Mathematics Subject Classification. Primary 58C40; Secondary 53C20.

Key words and phrases. Riemannian submanifolds, extrinsic balls, torsional rigidity, $L^{1}$-moment spectrum, mean exit time, isoperimetric inequalities, Green operator, Poincaré and Barta inequalities.

${ }^{\natural}$ Supported by the Spanish Mineco-FEDER grant MTM2010-21206-C02-01 and Junta de Andalucia grants FQM-325 and P09-FQM-5088.

\# * Supported by the Spanish Mineco-FEDER grant MTM2010-21206-C02-02 and by the Pla de Promoció de la Investigació de la Universitat Jaume I. 
special domains included in well known ambient spaces. On the other hand, the important challenge of finding upper and lower bounds of this first Dirichlet eigenvalue $\lambda_{1}(D)$ has received much attention by many authors since the classical works of Polya and Szegö [Po, PS].

In this paper we present an exact expression of the first Dirichlet eigenvalue of geodesic balls in general rotationally symmetric space in terms of the so-called mean exit time moment spectrum for these balls. This is obtained by applying a fundamental Green operator bootstrapping technique due to S. Sato, [Sa]. Moreover, using this expression and the comparison techniques developed in [HMP1] and [McMe], we then obtain upper and lower estimates for the first Dirichlet eigenvalue.

The mean exit time moment spectrum has been considered by P. McDonald in [Mc2, Mc1] for a quite similar purpose. Since the moment spectrum thus plays a key rôle and obviously contains a wealth of geometric information (as does the Dirichlet spectrum) we will briefly introduce it already here:

Let us consider the induced Brownian motion $X_{t}$ defined on the Riemannian manifold $(N, g)$. We consider the functions $u_{k}, k \geqslant 0$, defined inductively as the following sequence of solutions to a hierarchy of boundary value problems in $D \subset N$ : First we let

$$
u_{0}=1 \text { on } D \text {, }
$$

and then for $k \geqslant 1$ we define

$$
\begin{aligned}
\Delta u_{k}+k u_{k-1} & =0 \text { on } D, \\
\left.u_{k}\right|_{\partial D} & =0 .
\end{aligned}
$$

The first non-trivial function in this sequence, $u_{1}(x)$, is the mean time of first exit from $D$ for the Brownian motion starting at the point $x$ in $D$, see [Dy, $\mathrm{M}]$.

The $L^{1}$-moments of the exit time of $X_{t}$ from the smooth precompact domain $D$, also called the exit time moment spectrum of $D,\left\{\mathcal{A}_{k}(D)\right\}_{k=0}^{\infty}$, is then given by the following integrals, see [Mc1, Dy]:

$$
\mathcal{A}_{k}(D)=\int_{D} u_{k}(x) d V
$$

In particular, the quantity $\mathcal{A}_{1}(D)$ is known as the torsional rigidity of $D$. This name stems from the fact that if $D \subset \mathbb{R}^{2}$, then $\mathcal{A}_{1}(D)$ represents the torque required per unit angle of twist and per unit beam length when twisting an elastic beam of uniform cross section $D$, see $[\mathrm{Ba}]$ and $[\mathrm{PS}]$.

In the spirit of several previous seminal works, see e.g. [Ch1, Ch2] and [BBC, VBG], we investigate to what extent the $L^{1}$-moment spectrum associated to the domain $D \subset N$ can replace, support, or estimate its corresponding Dirichlet spectrum in order to establish good descriptors for the geometry of $D$ and, eventually, for the geometry of the manifold $N$.

The first direct eigenvalue comparison findings in this direction are due to P. McDonald and R. Meyers, [Mc2, Mc1, McMe]. As applied in [McMe], we shall also use the following observation as another benchmark strategy for the results reported in the present paper, see Subsection 4.2 . 
Theorem 1.1. The first Dirichlet eigenvalue $\lambda_{1}(D)$ of any smooth domain $D \subset N^{n}$ can be directly extracted from the corresponding exit time moment spectrum $\left\{\mathcal{A}_{k}(D)\right\}_{k=k_{0}}^{\infty}$ as follows:

$$
\lambda_{1}(D)=\sup \left\{\eta \geqslant 0: \lim _{n \rightarrow \infty} \sup \left(\frac{\eta}{2}\right)^{n} \frac{\mathcal{A}_{n}(D)}{\Gamma(n+1)}<\infty\right\} .
$$

Here we restrict our studies to be concerned with the exit time moment spectra and the first Dirichlet eigenvalues of a specific kind of domains: Firstly the geodesic $R$-balls in rotationally symmetric (warped product) model spaces $M_{w}^{m}$ and secondly the so-called extrinsic $R$-balls $D_{R} \subset P^{m}$ of properly immersed submanifolds $P^{m}$ in ambient Riemannian manifolds $N^{n}$ with controlled sectional curvatures and possessing at least one pole $p \in N^{n}$, see [S].

We are going to introduce in the following two subsections of this Introduction the contributions we have done in this work concerning the two problems above alluded.

1.1. The first eigenvalue of geodesic balls in rotationally symmetric manifolds. The computation of the first Dirichlet eigenvalue of a geodesic ball $B_{r}^{b, n}$ in the simply connected real space forms $\mathbb{K}^{n}(b)$ of constant curvature $b$ is well known in the case $b=0$, where $\lambda_{1}\left(B_{r}^{0, n}\right)=\left(\frac{c(n)}{r}\right)^{2}$ holds. Here $c(n)$ denotes the first zero of the Bessel function of order equal to $n / 2-1$. However, much less is known when the ambient space is the Hyperbolic space or the Sphere. In dimension 3, Savo shows in [Sav] that $\lambda_{1}\left(B_{r}^{b, 3}\right)=-b+\pi^{2} / r^{2}$ (see also $[\mathrm{FH}]$ for the corresponding result in $\mathrm{S}^{3}$ ). For the general dimensional case, we only have upper and lower bounds for $\lambda_{1}\left(B_{r}^{-1, n}\right)$ (see e.g. [G, Y, Ch1, BM, Sav]) and for $b=1$ the only known values are $\lambda_{1}\left(B_{\pi / 2}^{1, n}\right)=n$ and $\lambda_{1}\left(B_{\pi}^{1, n}\right)=0$. In the latter case, we can also find estimates (specially for $n=2$ ) in [Sa, MaT, Pi, Ch1, BCG, BaBe1]. Applying Cheng's eigenvalue comparison, we also have the upper bound $\lambda_{1}\left(B_{r}^{1, n}\right)<\left(\frac{c(n)}{r}\right)^{2}$. There is large literature in this subject, we just give here some selected references.

The first main result in this paper is a precise expression of the first Dirichlet eigenvalue of a geodesic $R$-ball in a rotationally symmetric model space $M_{w}^{m}$, which is defined as the warped product manifold $\left[0, \infty\left[\times_{w} S_{1}^{m-1}\right.\right.$ with a pole $p$ (see Definition 2.4 below). This expression, given in terms of its mean exit time moment spectrum implies an estimation for the first Dirichlet eigenvalue of a geodesic ball in the rotationally symmetric spaces, including the real space forms of constant curvature. Indeed, we will see that

$$
\frac{k u_{k-1}(0)}{u_{k}(0)} \leqslant \lambda_{1}\left(B_{R}^{w}\right) \leqslant \frac{k \mathcal{A}_{k-1}\left(B_{R}^{w}\right)}{\mathcal{A}_{k}\left(B_{R}^{w}\right)},
$$

for all $k \geqslant 1$ (see (1.3) and (1.4)), where the above bounds improve as $k$ increases. We emphasize that the exact value $\lambda_{1}\left(B_{R}^{w}\right)$ is obtained in the limit.

Theorem A. Let $B_{R}^{w}(p)$ be the geodesic ball of radius $R$ centered at the pole $p$ in $M_{w}^{m}$. Then the first eigenvalue of the ball can be expressed as the following limits of exit time moment data:

$$
\lambda_{1}\left(B_{R}^{w}\right)=\lim _{k \rightarrow \infty} \frac{k u_{k-1}(0)}{u_{k}(0)}=\lim _{k \rightarrow \infty} \frac{k \mathcal{A}_{k-1}\left(B_{R}^{w}\right)}{\mathcal{A}_{k}\left(B_{R}^{w}\right)},
$$

where $u_{k}$ are the solutions of the boundary value problem (1.3) defined on the geodesic $R$-ball $B_{R}^{w}$, and $\mathcal{A}_{k}\left(B_{R}^{w}\right)$ is the corresponding $k$-moment of $B_{R}^{w}$. Moreover, the radial function $g_{\infty}(r):=$ $\lim _{k \rightarrow \infty} \frac{u_{k}(r)}{u_{k}(0)}$ is a Dirichlet eigenfunction for the first eigenvalue $\lambda_{1}\left(B_{R}^{w}\right)$. 
In Section 3, as application of this theorem, we will derive several estimates of $\lambda_{1}$ for geodesic balls in the Sphere $S^{5}$ computing the function $u_{k}$ for some values of $k$.

On the other hand and as a consequence of Theorem A, we prove in Section 4 upper and lower bounds of the first Dirichlet eigenvalue of extrinsic balls in a submanifold together with intrinsic versions of these results and under a more relaxed set of curvature conditions.

1.2. A first glimpse of the comparison results. As we have previously mentioned, another important problem concerning the first Dirichlet eigenvalue consists in to find upper and lower bounds of it.

Concerning upper estimates, the classical and well-known comparison theorem by S. Y. Cheng, [Chg2], gives a sharp upper bound for the first eigenvalue in terms of the diameter for Riemannian manifolds with Ricci curvatures bounded from below.

Cheng also proved a lower bound in [Chg1] via a comparison theorem for the first eigenvalue of a geodesic ball in a complete Riemannian manifold with sectional curvatures bounded from above by a given constant. If this bounding constant is negative, then we also have the lower bound for the fundamental tone of a Cartan-Hadamard manifold established by H. P. McKean in [McK].

McKean's inequality was later generalized by L. F. Cheung and P. F. Leung in [CL] to the setting of complete non-compact submanifolds with bounded mean curvatures in hyperbolic spaces with constant negative curvature.

Using Theorem A and the comparison techniques and results developed in [HMP1] we then obtain upper and lower estimates for the first Dirichlet eigenvalue of the extrinsic balls of a submanifold in a more general setting and, as a corollary we recover McKean's result and obtain also upper and lower bounds for the fundamental tone of a Cartan-Hadamard manifold and the fundamental tone of a submanifold with controlled mean curvature along the lines of Cheung-Leung in [CL] and Bessa-Montenegro in [BM].

On the other hand we also show how the techniques developed in the papers [McMe], [HMP1] and [HMP2] can be applied, using Theorem 1.1 to give alternative proofs for some of these comparison results, see Subsection 4.2.

In order to illustrate our use of the upper and lower bounds on the ambient space sectional curvatures in the more general settings we extract here some consequences of Theorems 4.1 and 4.2, under some specific restrictive assumptions that are actually not needed for the general versions of the theorems. The first of these results is a lower bound for the first Dirichlet eigenvalue of extrinsic balls in a submanifold $P^{m}$ with controlled mean curvature $H_{P}$ in a Cartan-Hadamard manifold $N^{n}$.

To describe properly this control on the mean curvature, we need the following definition:

Definition 1.2. Let us consider a pole $p$ in the ambient Cartan-Hadamard manifold $N$. The $p$-radial mean curvature function for $P^{m}$ in $N^{n}$ is defined in terms of the inner product of $H_{P}$ with the $N$-gradient of the distance function $r(x)$ from the pole $p$ as follows:

$$
\mathcal{C}(x)=-\left\langle\nabla^{N} r(x), H_{P}(x)\right\rangle \text { for all } x \in P .
$$

Using a suitable radial control on the function $\mathcal{C}(x)$ we then obtain:

Theorem B. Let $N^{n}$ be a Cartan-Hadamard manifold, with sectional curvatures bounded from above by a constant $K_{N} \leqslant b \leqslant 0$. Let $p \in N$ be a pole in $N$. Let $P^{m} \subseteq N^{n}$ be a complete and non-compact properly immersed submanifold with p-radial mean curvature function $\mathcal{C}(x) \leqslant$ 
$h(r(x))$ for all $x \in P^{m}$, where $h(r)$ is a radial smooth function, called a radial bounding function from above. Suppose that

$$
(m-1) \cdot \sqrt{-b} \operatorname{coth}(R \sqrt{-b}) \geqslant m \cdot \sup _{r \in[0, R]} h(r),
$$

where we read $\sqrt{-b} \operatorname{coth}(R \sqrt{-b})$ to be $1 / R$ when $b=0$.

For any given extrinsic ball $D_{R}(p)$ in $P^{m}$ we then have the following inequality:

$$
\lambda_{1}\left(D_{R}\right) \geqslant \frac{1}{4}\left((m-1) \cdot \sqrt{-b} \operatorname{coth}(R \sqrt{-b})-m \cdot \sup _{r \in[0, R]} h(r)\right)^{2} .
$$

The radial bounding function $h(r)$ is related to the global extrinsic geometry of the submanifold. For example, it is obvious that minimal submanifolds $P^{m}$ satisfy that $\mathcal{C}(x)=$ 0 for all $x \in P^{m}$, with bounding function $h=0$. On the other hand, it can be proved, see the works [Sp, DCW, Pa, MP3], that when the submanifold is a convex hypersurface, then we have $\mathcal{C}(x) \geqslant 0$ for all $x \in P^{m}$ so the constant function $h(r)=0$ is thence a radial bounding function from below.

We obtain McKean's result from these theorems concerning the intrinsic situation, assuming that $P^{m}=N^{n}$. In this case the extrinsic domains $D_{R}$ become the geodesic balls $B_{R}^{N}$ of $N^{n}$ as we have pointed out above and we have moreover that $H_{P}(x)=0$ for all $x \in P^{m}$, so we consider the bound $h(r)=0$ for all $r>0$. Hence we obtain for $b<0$ and for all $R>0$ :

$$
\lambda_{1}\left(B_{R}^{N}\right) \geqslant \frac{1}{4}((n-1) \sqrt{-b} \operatorname{coth}(\sqrt{-b} R))^{2} .
$$

For $b \leqslant 0$ (we read $\sqrt{-b} \operatorname{coth}(R \sqrt{-b})$ to be $1 / R$ when $b=0$ ) we therefore have

$$
\lambda^{*}(N)=\lim _{R \rightarrow \infty} \lambda_{1}\left(B_{R}^{N}\right) \geqslant \frac{(n-1)^{2}|b|}{4} .
$$

We note that G. P. Bessa and J. F. Montenegro observe in [BM] an improvement of the bound (1.7) in the intrinsic setting as follows:

Theorem C (Bessa and Montenegro). Under the intrinsic conditions with $N^{n}$ having sectional curvatures bounded from above by $b \leqslant 0$ :

$$
\lambda_{1}\left(B_{R}^{N}\right) \geqslant \frac{1}{4}\left(\max \left(\frac{n}{R},(n-1) \cdot \sqrt{-b} \operatorname{coth}(R \sqrt{-b})\right)\right)^{2} .
$$

As a consequence of Theorem B, we also have a generalization of Theorem 2 in CheungLeung's paper [CL] and Corollary 4.4 in [BM]. The ambient manifold may in our case be a general Cartan-Hadamard manifold $N$ with sectional curvatures bounded from above by a constant $b<0$, (as in [BM], while [CL] considers the hyperbolic space $\mathbb{H}^{n}(-1)$ ), and our submanifold has radial mean curvature bounded by a radial function $h(r)$. This hypothesis includes complete and non-compact submanifolds $P^{m}$ in $N^{n}$ with mean curvature $H_{P}$ satisfying $\left\|H_{P}\right\| \leqslant \frac{\alpha}{m}$ with $\alpha \leqslant(m-1) \sqrt{-b}$, as in Cheung-Leung's and Bessa-Montenegro's statements, which are then generalized as follows:

$$
\lambda^{*}\left(P^{m}\right)=\lim _{R \rightarrow \infty} \lambda_{1}\left(B_{R}\right) \geqslant \frac{1}{4}((m-1) \sqrt{-b}-\alpha)^{2} .
$$

As an application of the proofs and techniques developed here we also obtain, when we consider a Riemannian manifold with sectional curvatures bounded from above by the corresponding sectional curvatures of a rotationally symmetric model space $M_{w}^{n}$, a direct first eigenvalue comparison result as follows: 
Theorem D. Let $B_{R}^{N}(p)$ be a geodesic ball of a complete Riemannian manifold $N^{n}$ with a pole $p$ and suppose that the $p$-radial sectional curvatures of $N^{n}$ are bounded from above by the $p_{w^{-}}$-radial sectional curvatures of a $w$-model space $M_{w}^{n}$. Then

$$
\lambda_{1}\left(B_{R}^{N}\right) \geqslant \lambda_{1}\left(B_{R}^{w}\right)
$$

where $B_{R}^{w}$ is the geodesic ball in $M_{w}^{n}$.

As already mentioned we observe in Section 4, that this result can also be obtained via the description given in [McMe] of the first Dirichlet eigenvalue of a domain $D$ in a Riemannian manifold in terms of its exit time moment spectrum, i.e. Theorem 1.1, in combination with the isoperimetric type inequalities for the exit time moment spectra established in [HMP2]. In the works [Mc1, Mc2] McDonald considers a complete Riemannian manifold $N$ which satisfies the following moment comparison condition with respect to a constant curvature space form $\mathbb{K}^{n}(b)$ : For every smooth bounded precompact domain $D \subset N$ and for all $k \in \mathbb{N}$, assume that $\mathcal{A}_{k}(D) \leqslant \mathcal{A}_{k}\left(B_{T}^{b, n}\right)$, where $B_{T}^{b, n}$ is a geodesic ball in $\mathbb{K}^{n}(b)$ such that $\operatorname{Vol}(D)=\operatorname{Vol}\left(B_{T}^{b, n}\right)$. Then $\lambda_{1}(D) \geqslant \lambda_{1}\left(B_{T}^{b, n}\right)$.

Using the same two-ways strategies we also obtain upper bounds for the first Dirichlet eigenvalue of intrinsic balls as follows:

Theorem E. Let $B_{R}^{N}$ be a geodesic ball of a complete Riemannian manifold $N^{n}$ with a pole $p$ and suppose that the p-radial sectional curvatures of $N^{n}$ are bounded from below by the $p_{w}$-radial sectional curvatures of a $w$-model space $M_{w}^{n}$. Then

$$
\lambda_{1}\left(B_{R}^{N}\right) \leqslant \lambda_{1}\left(B_{R}^{w}\right)
$$

where $B_{R}^{w}$ is the geodesic ball in $M_{w}^{n}$.

We remark that we also consider and prove extrinsic generalizations of this result using somewhat more elaborate comparison constellations which will be defined in Subsection 2.5, see Theorems 4.1 and 4.3 in Subsections 4.1 and 4.2. Moreover, in the intrinsic context given in Theorems $\mathrm{D}$ and $\mathrm{E}$, the equality with the bound is characterized under some specific condition satisfied by the model space $M_{w}^{n}$ which serve as a curvature-controller, (see Theorems 4.5 and 4.6 in Subsection 4.3).

1.3. Outline of the paper. Section 2 below is devoted to present all the preliminary concepts and instrumental prerequisites that we need in the following Sections 3 and 4 and which has not been introduced already. Theorem A is proved in Section 3. The general and complete versions of Theorems B, D, and E are then presented and proved in Section 4.

\section{Preliminaries And Comparison Settings}

We first consider a few conditions and concepts that will be instrumental for establishing our results in a more general version than presented in the introduction.

2.1. The extrinsic balls and the curvature bounds. We consider a properly immersed $m$ dimensional submanifold $P^{m}$ in a complete Riemannian manifold $N^{n}$. Let $p$ denote a point in $P^{m}$ and assume that $p$ is a pole of the ambient manifold $N$. We denote the distance function from $p$ in $N^{n}$ by $r(x)=\operatorname{dist}_{N}(p, x)$ for all $x \in N$. Since $p$ is a pole there is - by definition - a unique geodesic from $x$ to $p$ which realizes the distance $r(x)$. We also denote by $r$ the restriction $\left.r\right|_{P}: P^{m} \longrightarrow \mathbb{R}_{+} \cup\{0\}$. This restriction is then called the extrinsic distance function from $p$ in $P^{m}$. The corresponding extrinsic metric balls of (sufficiently large) radius $R$ 
and center $p$ are denoted by $D_{R}(p) \subset P^{m}$ and defined as any connected component which contains $p$ of the set:

$$
D_{R}(p)=B_{R}(p) \cap P=\{x \in P \mid r(x)<R\} ，
$$

where $B_{R}(p)$ denotes the geodesic $R$-ball around the pole $p$ in $N^{n}$. The extrinsic ball $D_{R}(p)$ is a connected domain in $P^{m}$, with boundary $\partial D_{R}(p)$. Since $P^{m}$ is assumed to be unbounded in $N$ we have for every sufficiently large $R$ that $B_{R}(p) \cap P \neq P$.

We now present the curvature restrictions which constitute the geometric framework of our investigations.

Definition 2.1. Let $p$ be a point in a Riemannian manifold $M$ and let $x \in M-\{p\}$. The sectional curvature $K_{M}\left(\sigma_{x}\right)$ of the two-plane $\sigma_{x} \in T_{x} M$ is then called a p-radial sectional curvature of $M$ at $x$ if $\sigma_{x}$ contains the tangent vector to a minimal geodesic from $p$ to $x$. We denote these curvatures by $K_{p, M}\left(\sigma_{x}\right)$.

Another notion needed to describe our comparison setting is the idea of radial tangency. If we denote by $\nabla r$ and $\nabla^{P} r$ the gradients of $r$ in $N$ and $P$ respectively, then we have the following basic relation:

$$
\nabla r=\nabla^{P} r+(\nabla r)^{\perp},
$$

where $(\nabla r)^{\perp}(q)$ is perpendicular to $T_{q} P$ for all $q \in P$.

When the submanifold $P$ is totally geodesic, then $\nabla r=\nabla^{P} r$ in all points, and, hence, $\left\|\nabla^{P} r\right\|=1$. On the other hand, and given the starting point $p \in P$, from which we are measuring the distance $r$, we know that $\nabla r(p)=\nabla^{P} r(p)$, so $\left\|\nabla^{P} r(p)\right\|=1$. Therefore, the difference $1-\left\|\nabla^{P} r\right\|$ quantifies the radial detour of the submanifold with respect the ambient manifold as seen from the pole $p$. To control this detour locally, we apply the following

Definition 2.2. We say that the submanifold $P$ satisfies a radial tangency condition at $p \in P$ when we have a smooth positive function

$$
g: P \mapsto \mathbb{R}_{+},
$$

so that

$$
\mathcal{T}(x)=\left\|\nabla^{P} r(x)\right\| \geqslant g(r(x))>0 \text { for all } x \in P .
$$

Remark 2.3. Of course, we always have

$$
\mathcal{T}(x)=\left\|\nabla^{P} r(x)\right\| \leqslant 1 \quad \text { for all } \quad x \in P .
$$

2.2. Model Spaces. As mentioned previously, the model spaces $M_{w}^{m}$ serve foremost as comparison controllers for the radial sectional curvatures of $N^{n}$.

Definition 2.4. [See [Gri], [GreW], [Pe]] A $w$-model $M_{w}^{m}$ is a smooth warped product with base $B^{1}=\left[0, R\left[\subset \mathbb{R}\right.\right.$ (where $0<R \leqslant \infty$ ), fiber $F^{m-1}=S_{1}^{m-1}$ (i.e. the unit $(m-1)$-sphere with standard metric), and warping function $w:\left[0, R\left[\rightarrow \mathbb{R}_{+} \cup\{0\}\right.\right.$ with $w(0)=0, w^{\prime}(0)=1, w^{(k)}(0)=0$ for all even derivation orders $k$ and $w(r)>0$ for all $r>0$. The point $p_{w}=\pi^{-1}(0)$, where $\pi$ denotes the projection onto $B^{1}$, is called the center point of the model space. If $R=\infty$, then $p_{w}$ is a pole of $M_{w}^{m}$. 
Remark 2.5. The simply connected space forms $\mathbb{K}^{m}(b)$ of constant curvature $b$ can be constructed as $w$-models with any given point as center point using the warping functions

$$
w(r)=w_{b}(r)=\left\{\begin{array}{ll}
\frac{1}{\sqrt{b}} \sin (\sqrt{b} r) & \text { if } b>0 \\
r & \text { if } b=0 \\
\frac{1}{\sqrt{-b}} \sinh (\sqrt{-b} r) & \text { if } b<0
\end{array} .\right.
$$

Note that for $b>0$ the function $w_{b}(r)$ admits a smooth extension to $r=\pi / \sqrt{b}$. For $b \leqslant 0$ any center point is a pole.

In the papers [O'N, GreW, Gri, MP1, MP2], we have a complete description of these model spaces, including the computation of their sectional curvatures $K_{p_{w}, M_{w}}$ in the radial directions from the center point. They are determined by the radial function $K_{p_{w}, M_{w}}\left(\sigma_{x}\right)=$ $K_{w}(r)=-\frac{w w^{\prime \prime}(r)}{w(r)}$. Moreover, the mean curvature of the distance sphere of radius $r$ from the center point is

$$
\eta_{w}(r)=\frac{w^{\prime}(r)}{w(r)}=\frac{d}{d r} \ln (w(r))
$$

2.3. The Isoperimetric Comparison Space. Given the bounding functions $g(r), h(r)$ and the ambient curvature controller function $w(r)$ described is Subsections 2.1 and 2.2, as in [MP3, HMP1] we construct a new model space $C_{w, g, h}^{m}$. For completeness, we recall this construction:

Definition 2.6. Given a smooth positive function

$$
g: P \mapsto \mathbb{R}_{+},
$$

satisfying $g(0)=1$ and $g(r(x)) \leqslant 1$ for all $x \in P$, a 'stretching' function $s$ is defined as follows

$$
s(r)=\int_{0}^{r} \frac{1}{g(t)} d t .
$$

It has a well-defined inverse $r(s)$ for $s \in[0, s(R)]$ with derivative $r^{\prime}(s)=g(r(s))$. In particular $r^{\prime}(0)=g(0)=1$.

Definition 2.7 ([MP3]). The isoperimetric comparison space $C_{w, g, h}^{m}$ is the $W$-model space with base interval $B=[0, s(R)]$ and warping function $W(s)$ defined by

$$
W(s)=\Lambda^{\frac{1}{m-1}}(r(s)),
$$

where the auxiliary function $\Lambda(r)$ satisfies the following differential equation:

$$
\begin{aligned}
\frac{d}{d r}\{\Lambda(r) w(r) g(r)\} & =\Lambda(r) w(r) g(r)\left(\frac{m}{g^{2}(r)}\left(\eta_{w}(r)-h(r)\right)\right) \\
& =m \frac{\Lambda(r)}{g(r)}\left(w^{\prime}(r)-h(r) w(r)\right),
\end{aligned}
$$

and the following boundary condition:

$$
\left.\frac{d}{d r}\right|_{r=0}\left(\Lambda^{\frac{1}{m-1}}(r)\right)=1 .
$$

We observe, that in spite of its relatively complicated construction, $C_{w, g, h}^{m}$ is indeed a model space $M_{W}^{m}$ with a well defined pole $p_{W}$ at $s=0: W(s) \geqslant 0$ for all $s$ and $W(s)$ is only 0 at $s=0$, where also, because of the explicit construction in definition 2.7 and because of 
equation (2.9): $W^{\prime}(0)=1$, and moreover, $W^{(k)}(0)=0$ for all even $k$, (see [MP3]).

Note that, when $g(r)=1$ for all $r$ and $h(r)=0$ for all $r$, then the stretching function $s(r)=r$ and $W(s(r))=w(r)$ for all $r$, so $C_{w, g, h}^{m}$ becomes a model space with warping function $w, M_{w}^{m}$.

These are the spaces where the bounds on the $L^{1}$-moment spectrum are attained. We shall refer to the $W$-model spaces $M_{W}^{m}=C_{w, g, h}^{m}$ as the isoperimetric comparison spaces of dimension $m$ defined by the radial functions $w, g$, and $h$.

2.4. Balance conditions. In the paper [HMP1] we imposed a balance condition on the general model spaces $M_{W}^{m}$, that we will need in the sequel:

Definition 2.8. The model space $M_{W}^{m}=C_{w, g, h}^{m}$ is $w$-balanced from below (with respect to the intermediary model space $M_{w}^{m}$ ) if the following holds for all $r \in[0, R]$, resp. all $s \in[0, s(R)]:$

$$
q_{W}(s)\left(\eta_{w}(r(s))-h(r(s))\right) \geqslant g(r(s)) / m .
$$

Here $q_{W}(s)$ is the isoperimetric quotient function

$$
\begin{aligned}
q_{W}(s) & =\frac{\operatorname{Vol}\left(B_{s}^{W}\right)}{\operatorname{Vol}\left(S_{s}^{W}\right)} \\
& =\frac{\int_{0}^{s} W^{m-1}(t) d t}{W^{m-1}(s)} \\
& =\frac{\int_{0}^{r(s)} \frac{\Lambda(u)}{g(u)} d u}{\Lambda(r(s))} .
\end{aligned}
$$

Remark 2.9. In particular the $w$-balance condition from below for $M_{W}^{m}=C_{w, g, h}^{m}$ implies that

$$
\eta_{w}(r)-h(r)>0 .
$$

Remark 2.10. The definition of $w$-balance condition from below for $M_{W}^{m}$ is clearly an extension of the balance condition from below as defined in [MP2, Definition 2.12]. The condition in that paper is obtained precisely when $g(r)=1$ and $h(r)=0$ for all $r \in[0, R]$ so that $r(s)=s, W(s)=w(r)$, and

$$
q_{w}(r) \eta_{w}(r) \geqslant 1 / m .
$$

In particular, the Hyperbolic spaces $\mathbb{H}^{n}(b)$ are balanced from below.

2.5. Comparison Constellations. We now present the precise settings where our main results take place, introducing the notion of comparison constellations as it was previously defined in [HMP1]. For that purpose we shall bound the previously introduced notions of radial curvature and tangency by the corresponding quantities attained in some special model spaces, called isoperimetric comparison spaces to be defined in the next subsection.

Definition 2.11. Let $N^{n}$ denote a complete Riemannian manifold with a pole $p$ and distance function $r=r(x)=\operatorname{dist}_{N}(p, x)$. Let $P^{m}$ denote an unbounded complete and closed submanifold in $N^{n}$. Suppose $p \in P^{m}$ and suppose that the following conditions are satisfied for all $x \in P^{m}$ with $r(x) \in[0, R]$ : 
(1) The $p$-radial sectional curvatures of $N$ are bounded from below by the $p_{w}$-radial sectional curvatures of of the $w$-model space $M_{w}^{m}$ :

$$
\mathcal{K}\left(\sigma_{x}\right) \geqslant-\frac{w^{\prime \prime}(r(x))}{w(r(x))} .
$$

(2) The $p$-radial mean curvature of $P$ is bounded from below by a smooth radial function $h(r)$ :

$$
\mathcal{C}(x) \geqslant h(r(x)) \text {. }
$$

(3) The submanifold $P$ satisfies a radial tangency condition at $p \in P$, with smooth positive function $g$ i.e. we have a smooth positive function

$$
g: P \mapsto \mathbb{R}_{+},
$$

such that

$$
\mathcal{T}(x)=\left\|\nabla^{P} r(x)\right\| \geqslant g(r(x))>0 \text { for all } x \in P .
$$

Let $C_{w, g, h}^{m}$ denote the $W$-model with the specific warping function $W: \pi\left(C_{w, g, h}^{m}\right) \rightarrow \mathbb{R}_{+}$constructed in Definition 2.7, (Subsection 2.3), via $w, g$, and $h$. Then the triple $\left\{N^{n}, P^{m}, C_{w, g, h}^{m}\right\}$ is called an isoperimetric comparison constellation bounded from below on the interval $[0, R]$.

A "constellation bounded from above" is given by the following dual setting, (with respect to the definition above), considering the special $W$-model spaces $C_{w, g, h}^{m}$ with $g=1$ :

Definition 2.12. Let $N^{n}$ denote a Riemannian manifold with a pole $p$ and distance function $r=r(x)=\operatorname{dist}_{N}(p, x)$. Let $P^{m}$ denote an unbounded complete and closed submanifold in $N^{n}$. Suppose the following conditions are satisfied for all $x \in P^{m}$ with $r(x) \in[0, R]$ :

(1) The $p$-radial sectional curvatures of $N$ are bounded from above by the $p_{w}$-radial sectional curvatures of the $w$-model space $M_{w}^{m}$ :

$$
\mathcal{K}\left(\sigma_{x}\right) \leqslant-\frac{w^{\prime \prime}(r(x))}{w(r(x))} .
$$

(2) The $p$-radial mean curvature of $P$ is bounded from above by a smooth radial function $h(r)$ :

$$
\mathcal{C}(x) \leqslant h(r(x)) \quad .
$$

Let $C_{w, 1, h}^{m}$ denote the $W$-model with the specific warping function $W: \pi\left(C_{w, 1, h}^{m}\right) \rightarrow \mathbb{R}_{+}$ constructed, (in the same way as in Definition 2.11 above), in Definition 2.7 via $w, g=1$, and $h$. Then the triple $\left\{N^{n}, P^{m}, C_{w, 1, h}^{m}\right\}$ is called an isoperimetric comparison constellation bounded from above on the interval $[0, R]$.

2.6. Laplacian Comparison. We begin this section recalling the following Laplacian comparison Theorem for manifolds with a pole (see [GreW, JK, MP1, MP2, MP3, MM] for more details).

Theorem 2.13. Let $N^{n}$ be a manifold with a pole $p$, let $M_{w}^{m}$ denote a $w$-model space with center $p_{w}$. Then we have the following dual Laplacian inequalities for modified distance functions:

(i) Suppose that every $p$-radial sectional curvature at $x \in N-\{p\}$ is bounded by the $p_{w}$-radial sectional curvatures in $M_{w}^{m}$ as follows:

$$
\mathcal{K}(\sigma(x))=K_{p, N}\left(\sigma_{x}\right) \geqslant-\frac{w^{\prime \prime}(r)}{w(r)} .
$$


Then we have for every smooth function $f(r)$ with $f^{\prime}(r) \leqslant 0$ for all $r$, (respectively $f^{\prime}(r) \geqslant$ 0 for all $r$ ):

$$
\begin{aligned}
\Delta^{P}(f \circ r) \geqslant(\leqslant) & \left(f^{\prime \prime}(r)-f^{\prime}(r) \eta_{w}(r)\right)\left\|\nabla^{P} r\right\|^{2} \\
& +m f^{\prime}(r)\left(\eta_{w}(r)+\left\langle\nabla^{N} r, H_{P}\right\rangle\right),
\end{aligned}
$$

where $H_{P}$ denotes the mean curvature vector of $P$ in $N$.

(ii) Suppose that every $p$-radial sectional curvature at $x \in N-\{p\}$ is bounded by the $p_{w}$-radial sectional curvatures in $M_{w}^{m}$ as follows:

$$
\mathcal{K}(\sigma(x))=K_{p, N}\left(\sigma_{x}\right) \leqslant-\frac{w^{\prime \prime}(r)}{w(r)} .
$$

Then we have for every smooth function $f(r)$ with $f^{\prime}(r) \leqslant 0$ for all $r$, (respectively $f^{\prime}(r) \geqslant$ 0 for all $r$ ):

$$
\begin{aligned}
\Delta^{P}(f \circ r) \leqslant(\geqslant) & \left(f^{\prime \prime}(r)-f^{\prime}(r) \eta_{w}(r)\right)\left\|\nabla^{P} r\right\|^{2} \\
& +m f^{\prime}(r)\left(\eta_{w}(r)+\left\langle\nabla^{N} r, H_{P}\right\rangle\right),
\end{aligned}
$$

where $H_{P}$ denotes the mean curvature vector of $P$ in $N$.

\section{THE FIRST EIGENVALUE AND MOMENT SPECTRA \\ OF GEODESIC BALLS IN MODEL SPACES}

The aim of this section is to obtain the first eigenvalue of a geodesic ball $B_{R}^{w}$, namely $\lambda_{1}\left(B_{R}^{w}\right)$, in terms of the $L^{1}$-moment spectrum of $B_{R}^{w}$.

To do that, we divide this section in two subsections. In the first, we shall provide a precise integral description of the $L^{1}$-moment spectrum of $B_{R}^{w}$ as previously also presented in [HMP1]). In the second we shall relate the first Dirichlet eigenvalue of the geodesic balls with its moment spectrum using a bootstrapping technique for the Green operator due to S. Sato, [Sa].

3.1. The moment spectrum of a geodesic model $R$-ball. We have the following result concerning the $L^{1}$-moment spectrum of a geodesic $R$-ball $B_{R}^{w} \subset M_{w}^{m}$, see [HMP1] for its proofs:

Proposition 3.1. Let $\tilde{u}_{k}$ be the solution of the boundary value problems (1.3), defined on the geodesic R-ball $B_{R}^{w}$ in a warped model space $M_{w}^{m}$.

Then

$$
\tilde{u}_{k}(r)=k \int_{r}^{R} \frac{\int_{0}^{t} w^{m-1}(s) \tilde{u}_{k-1}(s) d s}{w^{m-1}(t)} d t
$$

and

$$
\tilde{u}_{k}^{\prime}(r)=-k \frac{\int_{0}^{r} w^{m-1}(s) \tilde{u}_{k-1}(s) d s}{w^{m-1}(r)} .
$$

Therefore, applying the Divergence Theorem

$$
\mathcal{A}_{k}\left(B_{R}^{w}\right)=-\frac{1}{k+1} \tilde{u}_{k+1}^{\prime}(R) \operatorname{Vol}\left(S_{R}^{w}\right),
$$

where $S_{R}^{w}$ is the geodesic R-sphere in $M_{w}^{m}$. 
Let us consider now $M_{W}^{m}$ be an isoperimetric comparison model space and let $\tilde{u}_{k}^{W}$ be the radial functions given by (3.2), which are the solutions of the problems (1.3) defined on the geodesic ball $B_{s(R)}^{W}$. We define the functions $f_{k}:[0, R] \rightarrow \mathbb{R}$ as $f_{k}=\tilde{u}_{k}^{W} \circ s$, where $s$ is the stretching function given by (2.6). Then, we have the following

Lemma 3.2. Let $M_{W}^{m}$ be an isoperimetric comparison model space that is w-balanced from below in the sense of Definition 2.8. Then for all $k \geqslant 1$,

$$
f_{k}^{\prime \prime}(r)-f_{k}^{\prime}(r) \eta_{w}(r) \geqslant 0 .
$$

3.2. The first Dirichlet eigenvalue of geodesic balls in the model spaces $M_{w}^{m}$. Since $M_{w}^{m}$ is a spherically symmetric manifold with pole $p$, the Green operator for radial functions on $B_{R}^{w}(p)$ is given by

$$
G(u)(r)=\int_{r}^{R} \frac{\int_{0}^{t} w^{m-1}(s) u(s) d s}{w^{m-1}(t)} d t .
$$

Note that the moment functions $\tilde{u}_{k}$ given by (1.3), can be written via the Green operator as $\tilde{u}_{k}(r)=k G\left(\tilde{u}_{k-1}\right)(r)$. Using these functions, we can prove the following estimate

Proposition 3.3. Let $\tilde{u}_{k}$ be the radial functions defined on $D=B_{R}^{w}(p)$ and given by (1.3). Then, for all $k \geqslant 1$, the functions $\frac{k \tilde{u}_{k-1}}{\tilde{u}_{k}}(r)$ are increasing and we have

$$
\frac{k \tilde{u}_{k-1}}{\tilde{u}_{k}}(0) \leqslant \lambda_{1}\left(B_{R}^{w}\right) \leqslant \frac{k \mathcal{A}_{k-1}\left(B_{R}^{w}\right)}{\mathcal{A}_{k}\left(B_{R}^{w}\right)},
$$

where $\mathcal{A}_{k}\left(B_{R}^{w}\right)$ is the k-moment of $B_{R}^{w}$.

Proof. As $\tilde{u}_{k}$ is a positive radial $C^{2}$-function on $B_{R}^{w}$ satisfying that $\tilde{u}_{k}(R)=0$, a direct application of Barta's inequalities, (see [Bar] and [Ch1]) give us that

$$
\inf _{B_{R}^{w}}\left(\frac{k \tilde{u}_{k-1}}{\tilde{u}_{k}}(r)\right) \leqslant \lambda_{1}\left(B_{R}^{w}\right) \leqslant \sup _{B_{R}^{w}}\left(\frac{k \tilde{u}_{k-1}}{\tilde{u}_{k}}(r)\right), \quad \text { for all } k \geqslant 1 .
$$

Now, we are going to show inductively that the functions $k \tilde{u}_{k-1}(r) / \tilde{u}_{k}(r)$ are increasing for all $k \geqslant 1$ and consequently the value of the infimum and the supremum are attained in $r=0$ and $r=R$ respectively.

So, we begin studying the quotient $2 \tilde{u}_{1}(r) / \tilde{u}_{2}(r)$ (note that we can assume that $\tilde{u}_{0} \equiv 1$, and $1 / \tilde{u}_{1}(r)$ would be increasing). By (3.1) and (3.2) the first derivative of the quotient is given by

$$
\begin{aligned}
& \left(\frac{2 \tilde{u}_{1}}{\tilde{u}_{2}}\right)^{\prime}(r)=4 \frac{-G\left(\tilde{u}_{1}\right)(r) \int_{0}^{r} w^{m-1}(s) d s+G(1)(r) \int_{0}^{r} w^{m-1}(s) \tilde{u}_{1}(s) d s}{w^{m-1}(r)\left(\tilde{u}_{2}(r)\right)^{2}} \\
= & \frac{\int_{r}^{R} \frac{-4}{w^{m-1}(t)}\left(\int_{0}^{r} w^{m-1}(s) d s \int_{0}^{t} w^{m-1}(s) \tilde{u}_{1}(s) d s-\int_{0}^{t} w^{m-1}(s) d s \int_{0}^{r} w^{m-1}(s) \tilde{u}_{1}(s) d s\right) d t}{w^{m-1}(r)\left(\tilde{u}_{2}(r)\right)^{2}} .
\end{aligned}
$$

We define $h(r):=\frac{\int_{0}^{r} w^{m-1}(s) d s}{\int_{0}^{r} w^{m-1}(s) \tilde{u}_{1}(s) d s}$. Since $\tilde{u}_{1}$ is a decreasing function, 


$$
h^{\prime}(r)=\frac{w^{m-1}(r) \int_{0}^{r} w^{m-1}(s) \tilde{u}_{1}(s) d s-w^{m-1}(r) \tilde{u}_{1}(r) \int_{0}^{r} w^{m-1}(s) d s}{\left(\int_{0}^{r} w^{m-1}(s) \tilde{u}_{1}(s) d s\right)^{2}} \geqslant 0,
$$

and $h(r) \leqslant h(t)$ for all $t \geqslant r$. Therefore,

$$
\int_{0}^{r} w^{m-1}(s) d s \int_{0}^{t} w^{m-1}(s) \tilde{u}_{1}(s) d s-\int_{0}^{t} w^{m-1}(s) d s \int_{0}^{r} w^{m-1}(s) \tilde{u}_{1}(s) d s \leqslant 0,
$$

and $\left(\frac{2 \tilde{u}_{1}}{\tilde{u}_{2}}\right)^{\prime}(r) \geqslant 0$. Now, if we assume that $k \tilde{u}_{k-1} / \tilde{u}_{k}=\tilde{u}_{k-1} / G\left(\tilde{u}_{k-1}\right)$ is increasing, applying Lemma 2 of [Sa] we obtain that $(k+1) \tilde{u}_{k} / \tilde{u}_{k+1}=G\left(\tilde{u}_{k-1}\right) / G^{2}\left(\tilde{u}_{k-1}\right)$ is also increasing and we are done.

By virtue of the monoticity of the quotients $k \tilde{u}_{k-1}(r) / \tilde{u}_{k}(r)$ equation (3.6) reads

$$
\inf _{B_{R}^{w}}\left(\frac{k \tilde{u}_{k-1}}{\tilde{u}_{k}}(r)\right)=\frac{k \tilde{u}_{k-1}}{\tilde{u}_{k}}(0) \leqslant \lambda_{1}\left(B_{R}^{w}\right) \leqslant \sup _{B_{R}^{w w}}\left(\frac{k \tilde{u}_{k-1}}{\tilde{u}_{k}}(r)\right)=\frac{k \tilde{u}_{k-1}}{\tilde{u}_{k}}(R) .
$$

Notice that $\tilde{u}_{k}(R)=0$ for all $k \geqslant 1$, then by $\frac{k \tilde{u}_{k-1}}{\tilde{u}_{k}}(R)$ we mean,

$$
\lim _{r \rightarrow R} k \tilde{u}_{k-1}(r) / \tilde{u}_{k}(r)=\lim _{r \rightarrow R} \frac{k \tilde{u}_{k-1}^{\prime}(r)}{\tilde{u}_{k}^{\prime}(r)}=\frac{k \tilde{u}_{k-1}^{\prime}(R)}{\tilde{u}_{k}^{\prime}(R)} .
$$

To obtain (3.5) from inequalities (3.7), taking into account equation (3.3), for $k \geqslant 2$,

$$
\frac{k \tilde{u}_{k-1}^{\prime}(R)}{\tilde{u}_{k}^{\prime}(R)}=\frac{(k-1) \mathcal{A}_{k-2}\left(B_{R}^{w}\right)}{\mathcal{A}_{k-1}\left(B_{R}^{w}\right)} .
$$

When $k=1$, the above proposition gives us immediately the following upper and lower bounds for the first eigenvalue of a geodesic ball. The lower bound was obtained in [BCG] for geodesic balls in the $n$-dimensional sphere $S^{n}(1)$, and later it was generalized for an arbitrary $M_{w}^{m}$ in [BaBe2]. The upper bound give us a new relation between the first Dirichlet eigenvalue and the torsional rigidity of a geodesic ball in a rotationally symmetric space.

Corollary 3.4. The first eigenvalue $\lambda_{1}\left(B_{R}^{w}\right)$ of the geodesic balls in the rotationally symmetric spaces $M_{w}^{m}$ satisfies

$$
\frac{1}{\int_{0}^{R} q_{w}(t) d t} \leqslant \lambda_{1}\left(B_{R}^{w}\right) \leqslant \frac{\operatorname{vol}\left(B_{R}^{w}\right)}{\mathcal{A}_{1}\left(B_{R}^{w}\right)}
$$

where $q_{w}(t)$ is the isoperimetric quotient defined by

$$
q_{w}(t):=\frac{\int_{0}^{t} w^{m-1}(s) d s}{w^{m-1}(t)} .
$$

We are going now to show that Proposition 3.3 give us better estimations of the first Dirichlet eigenvalue of the geodesic balls in rotationally symmetric spaces, because inequalities (3.5) improve when $k$ increases.

Corollary 3.5. Let $\tilde{u}_{k}$ be the functions defined on $B_{R}^{w}$ and given by (3.1). Then,

$$
\lim _{k \rightarrow \infty} \frac{k \tilde{u}_{k-1}}{\tilde{u}_{k}}(0) \leqslant \lambda_{1}\left(B_{R}^{w}\right) \leqslant \lim _{k \rightarrow \infty} \frac{k \mathcal{A}_{k-1}\left(B_{R}^{w}\right)}{\mathcal{A}_{k}\left(B_{R}^{w}\right)} .
$$


Proof. Since $G$ is a positive operator and $(k+1) \tilde{u}_{k} / \tilde{u}_{k+1}=G\left(\tilde{u}_{k-1}\right) / G^{2}\left(\tilde{u}_{k-1}\right)$, applying Lemma 1 of [Sa], we obtain that for $k \geqslant 1$, and for all $r \in[0, R]$ :

$$
\inf _{B_{R}^{w w}}\left(\frac{k \tilde{u}_{k-1}}{\tilde{u}_{k}}(r)\right)=\frac{k \tilde{u}_{k-1}}{\tilde{u}_{k}}(0) \leqslant \frac{(k+1) \tilde{u}_{k}}{\tilde{u}_{k+1}}(r) \leqslant \sup _{B_{R}^{w}}\left(\frac{k \tilde{u}_{k-1}}{\tilde{u}_{k}}(r)\right)=\frac{k \tilde{u}_{k-1}}{\tilde{u}_{k}}(R) .
$$

In particular, for $r=0$ and for all $k \geqslant 1$, we have

$$
\frac{k \tilde{u}_{k-1}}{\tilde{u}_{k}}(0) \leqslant \frac{(k+1) \tilde{u}_{k}}{\tilde{u}_{k+1}}(0) \text {. }
$$

Moreover, by Proposition 3.3, we know that, for all $k \geqslant 1$, $\frac{k \tilde{u}_{k-1}}{\tilde{u}_{k}}(0) \leqslant \lambda_{1}\left(B_{R}^{w}\right)$, so therefore $\left\{\frac{k \tilde{u}_{k-1}}{\tilde{u}_{k}}(0)\right\}_{k=1}^{\infty}$ is a bounded increasing sequence, so there exists

$$
\lambda:=\lim _{k \rightarrow \infty} \frac{k \tilde{u}_{k-1}}{\tilde{u}_{k}}(0) \leqslant \lambda_{1}\left(B_{R}^{w}\right) .
$$

On the other hand, taking $r=R$ in (3.13) we obtain, for all $k \geqslant 1$

$$
\frac{(k+1) \tilde{u}_{k}}{\tilde{u}_{k+1}}(R) \leqslant \frac{k \tilde{u}_{k-1}}{\tilde{u}_{k}}(R) .
$$

Moreover, by inequality (3.7) in Proposition 3.3, we know that, for all $k \geqslant 1, \frac{k \tilde{u}_{k-1}}{\tilde{u}_{k}}(R) \geqslant$ $\lambda_{1}\left(B_{R}^{w}\right)$, so therefore $\left\{\frac{k \tilde{u}_{k-1}}{\tilde{u}_{k}}(R)\right\}_{k=1}^{\infty}$ is a decreasing sequence bounded from below with the limit

$$
\mu:=\lim _{k \rightarrow \infty} \frac{k \tilde{u}_{k-1}}{\tilde{u}_{k}}(R)=\lim _{k \rightarrow \infty} \frac{k \mathcal{A}_{k-1}\left(B_{R}^{w}\right)}{\mathcal{A}_{k}\left(B_{R}^{w}\right)} \geqslant \lambda_{1}\left(B_{R}^{w}\right),
$$

and this concludes the proof.

As a consequence of these results, for each $k \in \mathbb{N}$, we obtain the lower and upper bounds of $\lambda_{1}\left(B_{R}^{w}\right)$ given by (3.5). These bounds improve as $k$ increases; in particular we derive better estimates than those given in [BaBe2] and [BCG]. We will return to these facts at the end of this section.

Now, let us state and prove the main result of this section, which is also stated as Theorem $\mathrm{A}$ in the introduction:

Theorem 3.6. Let $B_{R}^{w}$ be the geodesic ball of radius $R$ in $M_{w}^{m}$. Then,

$$
\lambda_{1}\left(B_{R}^{w}\right)=\lim _{k \rightarrow \infty} \frac{k \tilde{u}_{k-1}(0)}{\tilde{u}_{k}(0)}=\lim _{k \rightarrow \infty} \frac{k \mathcal{A}_{k-1}\left(B_{R}^{w}\right)}{\mathcal{A}_{k}\left(B_{R}^{w}\right)},
$$

where $\tilde{u}_{k}$ are the functions defined by (3.1) and $\mathcal{A}_{k}\left(B_{R}^{w}\right)$ is the k-moment of $B_{R}^{w}$. Moreover, the radial $C^{2}$-function $g_{\infty}(r):=\lim _{k \rightarrow \infty} \frac{\tilde{u}_{k}(r)}{\tilde{u}_{k}(0)}$ is an eigenfunction of the first eigenvalue.

Proof. We are going to apply Barta's Lemma to show equality

$$
\lambda_{1}\left(B_{R}^{w}\right)=\lim _{k \rightarrow \infty} \frac{k \tilde{u}_{k-1}(0)}{\tilde{u}_{k}(0)} .
$$

For that, we shall first define (see Lemma 3.7 below) a positive radial $C^{2}$-function $g_{\infty}(r)$ defined on $B_{R}^{w}$ such that $g_{\infty}(R)=0$ and such that satisfies 


$$
\Delta g_{\infty}(r)=g_{\infty}^{\prime \prime}(r)+(m-1) \eta_{w}(r) g_{\infty}^{\prime}(r)=-\lambda g_{\infty}(r),
$$

where $\lambda=\lim _{k \rightarrow \infty} \frac{k \tilde{u}_{k-1}}{\tilde{u}_{k}}(0)$.

Then, $\lambda$ is a Laplacian eigenvalue on $B_{R}^{w}$ with eigenfunction $g_{\infty}(r)$. As $g_{\infty}(R)=0$, Barta's inequalities imply that

$$
\lambda=\inf _{B_{R}^{w}}\left(\frac{-\Delta g_{\infty}}{g_{\infty}}\right) \leqslant \lambda_{1}\left(B_{R}^{w}\right) \leqslant \sup _{B_{R}^{w}}\left(\frac{-\Delta g_{\infty}}{g_{\infty}}\right)=\lambda
$$

and hence

$$
\lambda=\lim _{k \rightarrow \infty} \frac{k \tilde{u}_{k-1}}{\tilde{u}_{k}}(0)=\lambda_{1}\left(B_{R}^{w}\right)
$$

We now need the following

Lemma 3.7. Let $g_{k}(r):=\tilde{u}_{k}(r) / \tilde{u}_{k}(0)$ be the functions defined by (3.1) normalized so that $g_{k}(0)=1$ for all $k$. Then $g_{\infty}(r):=\lim _{k \rightarrow \infty} g_{k}(r)$ is a positive radial $C^{2}$-function defined on $B_{R}^{w}$ such that $\Delta g_{\infty}(r)=-\lambda g_{\infty}(r)$ and $g_{\infty}(R)=0$.

Proof. Since $k \tilde{u}_{k-1}(r) / \tilde{u}_{k}(r)$ is increasing in $r$ for all $k$, we have that

$$
\frac{k \tilde{u}_{k-1}}{\tilde{u}_{k}}(r) \geqslant \frac{k \tilde{u}_{k-1}}{\tilde{u}_{k}}(0),
$$

and then, for a fixed $r,\left\{g_{k}(r)\right\}_{k=1}^{\infty}$ is a decreasing sequence of bounded functions converging pointwise to a function $g_{\infty}(r)$. Moreover, since $\tilde{u}_{k-1}$ is a decreasing function

$$
\left|g_{k}^{\prime}(r)\right|=\frac{k \int_{0}^{r} w^{m-1}(s) \tilde{u}_{k-1}(s) d s}{w^{m-1}(s) \tilde{u}_{k}(0)} \leqslant \frac{k \tilde{u}_{k-1}(0)}{\tilde{u}_{k}(0)} q_{w}(r) \leqslant \lambda \max _{[0, R]}\left\{q_{w}(r)\right\},
$$

where we have used that $\left\{k \tilde{u}_{k-1}(0) / \tilde{u}_{k}(0)\right\}_{k}$ is an increasing sequence converging to $\lambda$ and that $q_{w}(r)$ is the isoperimetric quotient defined in (3.11) that is a continuous function on the compact interval $[0, R]$.

Then, the first derivatives of the functions $g_{k}$ are uniformly bounded, and hence the sequence of functions is uniformly bounded and equicontinuous. As a consequence of the Ascoli-Arzela Theorem, $\left\{g_{k}\right\}_{k=1}^{\infty}$ converges to $g_{\infty}$ uniformly and $g_{\infty}$ is a continuous function. In addition,

$$
\begin{aligned}
\lim _{k \rightarrow \infty} g_{k}^{\prime}(r) & =\lim _{k \rightarrow \infty} \frac{-k \int_{0}^{r} w^{m-1}(s) \tilde{u}_{k-1}(s) d s}{w^{m-1}(r) u_{k}(0)} \\
& =\lim _{k \rightarrow \infty} \frac{-k \tilde{u}_{k-1}(0)}{\tilde{u}_{k}(0)} \frac{\int_{0}^{r} w^{m-1}(s) g_{k-1}(s) d s}{w^{m-1}(r)}=-\lambda \frac{\int_{0}^{r} w^{m-1}(s) g_{\infty}(s) d s}{w^{m-1}(r)} .
\end{aligned}
$$

Now, taking into account that

$$
\tilde{u}_{k}^{\prime \prime}(r)=-k \tilde{u}_{k-1}(r)-(m-1) \frac{w^{\prime}(r)}{w(r)} \tilde{u}_{k}^{\prime}(r),
$$

and using (3.18) and that $g_{k-1}$ is bounded above by 1 ,

$$
\begin{aligned}
\left|g_{k}^{\prime \prime}(r)\right| & =\left|-\frac{k \tilde{u}_{k-1}(0)}{\tilde{u}_{k}(0)} g_{k-1}(r)-(m-1) \eta_{w}(r) g_{k}^{\prime}(r)\right| \\
& \leqslant \lambda\left|g_{k-1}(r)\right|+(m-1) \lambda\left|\eta_{w}(r) q_{w}(r)\right| \\
& \leqslant \lambda+(m-1) \lambda\left|\eta_{w}(r) q_{w}(r)\right| .
\end{aligned}
$$


But $w(r)$ satisfies $w(0)=0$ and $w^{\prime}(0)=1$. Hence, $\eta_{w}(r) q_{w}(r)=\frac{w^{\prime}(r) \int_{0}^{r} w^{m-1}(t) d t}{w^{m}(r)}$ is a continuous function defined on $[0, R]$ because by L'Hopital rule

$$
\lim _{r \rightarrow 0} \frac{\int_{0}^{r} w^{m-1}(t) d t}{w^{m}(r)}=\frac{1}{m}
$$

Therefore, the derivatives $g_{k}^{\prime \prime}(r)$ are uniformly bounded on $[0, R]$ and the functions $g_{k}^{\prime}$ converge uniformly. As a consequence of this,

$$
g_{\infty}^{\prime}(r)=\lim _{k \rightarrow \infty} g_{k}^{\prime}(r)=-\lambda \frac{\int_{0}^{r} w^{m-1}(s) g_{\infty}(s) d s}{w^{m-1}(r)} .
$$

To finish the proof we only need to show that $g_{k}^{\prime \prime}(r)$ also converges uniformly to $g_{\infty}^{\prime \prime}(r)$. But it is clear using the definition of uniform convergence, since

$$
\begin{aligned}
\mid g_{k}^{\prime \prime}(r)+ & \lambda g_{\infty}(r)+(m-1) \eta_{w}(r) g_{\infty}^{\prime}(r) \mid= \\
& \left|-\frac{k \tilde{u}_{k-1}(0)}{\tilde{u}_{k}(0)} g_{k-1}(r)-(m-1) \eta_{w}(r) g_{k}^{\prime}(r)+\lambda g_{\infty}(r)+(m-1) \eta_{w}(r) g_{\infty}^{\prime}(r)\right| \\
\leqslant & \left|\lambda g_{\infty}(r)-\frac{k \tilde{u}_{k-1}(0)}{\tilde{u}_{k}(0)} g_{k-1}(r)\right| \\
& +(m-1)\left|\eta_{w}(r) \frac{\int_{0}^{r} w^{m-1}(s)\left(\frac{k \tilde{u}_{k-1}(0)}{\tilde{u}_{k}(0)} g_{k-1}(s)-\lambda g_{\infty}(s)\right) d s}{w^{m-1}(r)}\right| \\
\leqslant & \sup _{[0, R]}\left\{\left|\lambda g_{\infty}(r)-\frac{k \tilde{u}_{k-1}(0)}{\tilde{u}_{k}(0)} g_{k-1}(r)\right|\right\}\left(1+(m-1)\left|\eta_{w}(r) q_{w}(r)\right|\right) .
\end{aligned}
$$

We use the fact that the function $k \tilde{u}_{k-1}(0) / \tilde{u}_{k}(0) g_{k-1}(r)$ converges uniformly to $\lambda g_{\infty}(r)$ and that $\eta_{w}(r) q_{w}(r)$ is a continuous function defined on $[0, R]$. Then,

$$
g_{\infty}^{\prime \prime}(r)=-\lambda g_{\infty}(r)-(m-1) \eta_{w}(r) g_{\infty}^{\prime}(r) .
$$

The proof of the lemma is then finished by observing that $g_{k}$ are $C^{2}$-functions and that $g_{k}(R)=0$ for all $R$.

Returning to the proof of the Theorem, to show equality

$$
\lambda_{1}\left(B_{R}^{w}\right)=\lim _{k \rightarrow \infty} \frac{k \mathcal{A}_{k-1}\left(B_{R}^{w}\right)}{\mathcal{A}_{k}\left(B_{R}^{w}\right)},
$$

we argue in the same way, applying again Barta's Lemma using a positive $C^{2}$-function satisfying that $\Delta h_{\infty}(r)=-\mu h_{\infty}(r)$ and $h_{\infty}(R)=0$.

We need the following

Lemma 3.8. Let us define the functions $h_{k}(r)=\tilde{u}_{k}(r) /\left(-\tilde{u}_{k}^{\prime}(R)\right)$ for all $k \geqslant 0$. Then $h_{\infty}(r)=$ $\lim _{k \rightarrow \infty} h_{k}(r)$ is a positive radial $C^{2}$-function defined on $B_{R}^{w}$ such that $\Delta h_{\infty}(r)=-\mu h_{\infty}(r)$ and $h_{\infty}(R)=0$.

Proof. Let us normalize the functions $\tilde{u}_{k}$ by putting $h_{k}(r)=\tilde{u}_{k}(r) /\left(-\tilde{u}_{k}^{\prime}(R)\right)$. Then, since $k \tilde{u}_{k-1} / \tilde{u}_{k}$ is increasing for all $k$, we have that

$$
\frac{k \tilde{u}_{k-1}}{\tilde{u}_{k}}(r) \leqslant \frac{k \tilde{u}_{k-1}}{\tilde{u}_{k}}(R)=\frac{k \tilde{u}_{k-1}^{\prime}(R)}{\tilde{u}_{k}^{\prime}(R)},
$$


and $\left\{h_{k}(r)\right\}_{k=1}^{\infty}$ is an increasing sequence of functions. Moreover, $h_{k}(r) \leqslant h_{k}(0)=\tilde{u}_{k}(0) /\left(-\tilde{u}_{k}^{\prime}(R)\right)$ and

$$
\begin{aligned}
\frac{-\tilde{u}_{k}^{\prime}(R)}{\tilde{u}_{k}(0)} & =\frac{k \tilde{u}_{k-1}(0)}{\tilde{u}_{k}(0)} \frac{\int_{0}^{R} w^{m-1}(s) \frac{\tilde{u}_{k-1}(s)}{\tilde{u}_{k-1}(0)} d s}{w^{m-1}(R)} \\
& \geqslant \frac{1}{\tilde{u}_{1}(0)} \frac{\int_{0}^{R} w^{m-1}(s) g_{\infty}(s) d s}{w^{m-1}(R)}=\frac{-g_{\infty}^{\prime}(R)}{\lambda_{1} \tilde{u}_{1}(0)} .
\end{aligned}
$$

The functions $h_{k}$ are therefore bounded from above and thence they converge pointwise to a function $h_{\infty}$. Following the lines of the proof of lemma above and taking into account that $h_{k}(r)=\frac{\tilde{u}_{k}(0)}{-\tilde{u}_{k}^{\prime}(R)} g_{k}(r)$ and that $\frac{-\tilde{u}_{k}^{\prime}(R)}{\tilde{u}_{k}(0)}$ converges to $g_{\infty}^{\prime}(R)$ by (3.23), we can conclude that $h_{\infty}$ is a $C^{2}$-function satisfying that $h_{\infty}(R)=0$ and

$$
h_{\infty}^{\prime \prime}(r)=-\mu h_{\infty}(r)-(m-1) \eta_{w}(r) h_{\infty}^{\prime}(r) .
$$

This concludes the proof of Lemma 3.8.

To finish the proof of Theorem 3.6 we observe that, $h_{\infty}(r)=\lim _{k \rightarrow \infty} \frac{\tilde{u}_{k}(R)}{-\tilde{u}_{k}^{\prime}(R)}$ is a radial $C^{2}$-function on $B_{R}^{w}$ satisfying that $h_{\infty}(R)=0$. Moreover, $\Delta h_{\infty}=-\mu h_{\infty}$ by (3.24), where

$$
\mu:=\lim _{k \rightarrow \infty} \frac{k \mathcal{A}_{k-1}\left(B_{R}^{w}\right)}{\mathcal{A}_{k}\left(B_{R}^{w}\right)} .
$$

Again, Barta's inequalities give us the result.

Example. It is not very hard to compute explicitly the functions $\tilde{u}_{k}$ (given recursively by (1.3)) for some values of $k$ in $S^{2 m+1}(b)$ and $\mathbb{H}^{2 m+1}(b)$ to give upper and lower bounds for the first eigenvalue of a geodesic ball in terms of its radius (the expressions are not so nice as $k$ increases but one can use the help of a computer). To illustrate this, let us show some estimates in $S^{5}(1)$ (in the 3-dimensional sphere the exact value is known [FH]).

For $\mathbb{S}^{5}(1)$, we know that $w(r)=\sin (r)$ and the functions $\tilde{u}_{k}(r)$ for the geodesic $R$-ball can be computed using (3.1). By this way, we obtain that

$$
\begin{aligned}
\tilde{u}_{1}(0)= & \frac{1}{24}\left(5+3 \csc (R)^{2}-3 R \cot (R)\left(2+\csc (R)^{2}\right)\right) \\
\tilde{u}_{2}(0)= & \frac{1}{192}\left(-1-12 R^{2}-14 R \cot (R)-(5+19 R \cot (R)) \csc (R)^{2}\right. \\
\quad & \left.+6\left(1+3 R^{2}-2 R \cot (R)\right) \csc (R)^{4}+6 R^{2} \csc (R)^{6}\right), \\
\tilde{u}_{1}^{\prime}(R)= & -\frac{1}{32} \csc (R)^{4}(12 R-8 \sin (2 R)+\sin (4 R)), \\
\tilde{u}_{2}^{\prime}(R)= & \frac{1}{2048} \csc (R)^{7}\left(\left(-1+288 R^{2}\right) \cos (R)+3\left(9-32 R^{2}\right) \cos (3 R)\right. \\
& \quad-27 \cos (5 R)+\cos (7 R)-8 R(33 \sin (R)+5 \sin (3 R))),
\end{aligned}
$$

and the first eigenvalue satisfies

$$
\frac{2 \tilde{u}_{1}(0)}{\tilde{u}_{2}(0)} \leqslant \lambda_{1}\left(B_{R}^{\sin }\right) \leqslant \frac{2 \tilde{u}_{1}^{\prime}(R)}{\tilde{u}_{2}^{\prime}(R)} .
$$


We can compute recursively the following functions $\tilde{u}_{k}$ (the expressions become more and more involved). For $k=2$ and $k=5$ we show in table 1 the estimates of $\lambda_{1}\left(B_{R}^{\sin }\right)$ obtained for different radius of the geodesic ball using (3.5) and (3.9):

\begin{tabular}{|c|c|c|c|c|c|c|c|}
\hline $\mathrm{R}=$ & $\pi / 8$ & $\pi / 4$ & $3 \pi / 8$ & $\pi / 2$ & $5 \pi / 8$ & $3 \pi / 4$ & $7 \pi / 8$ \\
\hline$\lambda_{1} \geqslant 2 \tilde{u}_{1}(0) / \tilde{u}_{2}(0)$ & 99.015 & 23.336 & 9.2838 & 4.323 & 1.993 & 0.7408 & 0.1257 \\
\hline$\lambda_{1} \leqslant 2 \tilde{u}_{1}^{\prime}(R) / \tilde{u}_{2}^{\prime}(R)$ & 218.1651 & 48.2328 & 17.199 & 6.8572 & 2.6182 & 0.8152 & 0.12672 \\
\hline$\lambda_{1} \geqslant 5 \tilde{u}_{4}(0) / \tilde{u}_{5}(0)$ & 126.021 & 29.1441 & 11.2221 & 4.98633 & 2.1696 & 0.762655 & 0.12601996 \\
\hline$\lambda_{1} \leqslant 5 \tilde{u}_{4}^{\prime}(R) / \tilde{u}_{5}^{\prime}(R)$ & 128.718 & 29.6307 & 11.339 & 5.00926 & 2.1719 & 0.762702 & 0.12601997 \\
\hline
\end{tabular}

TABLE 1. Upper and lower estimates of $\lambda_{1}\left(B_{R}^{\sin }\right)$ in $S^{5}(1)$ for various values of $R$ and for $k=2$ and $k=5$.

For a greater value of $k$ we obtain better estimates. Anyway, our theorem asserts that in the limit $(k \rightarrow \infty)$ we obtain the exact value of the first eigenvalue.

\section{EXTRINSIC AND INTRINSIC EIGENVALUE COMPARISON THEOREMS}

In this section we now state and prove the comparison theorems for the first eigenvalue of extrinsic balls of submanifolds that we alluded to in the introduction, but here in the more general contexts of the comparison constellations. We divide this section into three parts.

In the first we use Theorem 3.6 to get upper and lower bounds for the first Dirichlet eigenvalues of the extrinsic balls of a submanifold.

In the second subsection we present the comparison between the first Dirichlet eigenvalues of the extrinsic balls and the geodesic balls with the same radius in the spaces used as a model and described in Subsection 4.1, but now the strategy is based on the description of the first Dirichlet eigenvalue of a domain in a Riemannian manifold given by McDonald and Myers in [McMe]. With this method it is furthermore possible to describe the equality case and obtain a rigidity result in one of the two settings studied.

The third subsection is devoted to an investigation of the intrinsic case, namely to apply the results obtained in Subsections 4.1 and 4.2 to the intrinsic geodesic balls of a manifold with sectional curvatures bounded from above or from below by the corresponding sectional curvatures of rotationally symmetric model spaces. In both of these (intrinsic) cases we also obtain the corresponding rigidity results.

\subsection{Extrinsic comparison. First strategy.}

Theorem 4.1. Let $\left\{N^{n}, P^{m}, C_{w, g, h}^{m}\right\}$ denote a comparison constellation bounded from below in the sense of Definition 2.11. Assume that $M_{W}^{m}=C_{w, g, h}^{m}$ is w-balanced from below. Let $D_{R}$ be a smooth precompact extrinsic $R$-ball in $P^{m}$ with center at a point $p \in P \subset N$ which also serves as a pole in $N$.

Then we have the following inequalities:

$$
\lambda_{1}\left(D_{R}\right) \leqslant \lambda_{1}\left(B_{s(R)}^{W}\right)=\lim _{k \rightarrow \infty} \frac{k \mathcal{A}_{k-1}\left(B_{s(R)}^{W}\right)}{\mathcal{A}_{k}\left(B_{S(R)}^{W}\right)} \leqslant \frac{\operatorname{Vol}\left(B_{S(R)}^{W}\right)}{\mathcal{A}_{1}\left(B_{S(R)}^{W}\right)},
$$

where $B_{s(R)}^{W}$ is the geodesic ball in $M_{W}^{m}$. 
Proof. Let $g_{\infty}(s)$ be the eigenfunction of $\lambda_{1}\left(B_{s(R)}^{W}\right)$ obtained in Theorem 3.6 and given by

$$
g_{\infty}(s)=\lim _{k \rightarrow \infty} \frac{\tilde{u}_{k}^{W}(s)}{\tilde{u}_{k}^{W}(0)} .
$$

If we compose $g_{\infty}$ with the stretching function defined by (2.6), we obtain a function $\tilde{g}$ : $[0, R] \rightarrow \mathbb{R}$ as

$$
\tilde{g}(r)=\lim _{k \rightarrow \infty} \frac{\tilde{u}_{k}^{W}(s(r))}{\tilde{u}_{k}^{W}(0)}=\lim _{k \rightarrow \infty} \frac{f_{k}(r)}{f_{k}(0)}
$$

where $f_{k}$ are the functions introduced in Lemma 3.2.

Now let $r$ denote the smooth distance to the pole $p$ on $N$. We define the radial function $v: D_{R} \rightarrow \mathbb{R}$ by $v(q)=\tilde{g}(r(q))$. Using Theorem 2.13 and the fact that

$$
\tilde{g}^{\prime}(r)=\lim _{k \rightarrow \infty} \frac{f_{k}^{\prime}(r)}{f_{k}(0)} \leqslant 0,
$$

since $f_{k}^{\prime} \leqslant 0$ for all $k$ and the convergence is uniform, we have that

$$
\Delta^{P} v=\Delta^{P}(\tilde{g} \circ r) \geqslant\left(\tilde{g}^{\prime \prime}(r)-\tilde{g}^{\prime}(r) \eta_{w}(r)\right)\left\|\nabla^{P} r\right\|^{2}+m \tilde{g}^{\prime}(r)\left(\eta_{w}(r)+\left\langle\nabla^{N} r, H_{P}\right\rangle\right)
$$

Again by the uniform convergence proved in Lemma 3.7,

$$
\tilde{g}^{\prime \prime}(r)-\tilde{g}^{\prime}(r) \eta_{w}(r)=\lim _{k \rightarrow \infty} \frac{f_{k}^{\prime \prime}(r)-\eta_{w}(r) f_{k}^{\prime}(r)}{f_{k}(0)} \geqslant 0,
$$

by virtue of Lemma 3.2.

Then, if we interchange the limits and the derivatives and use Lemma 3.2,

$$
\begin{aligned}
\Delta^{P} v=\Delta^{P}(\tilde{g} \circ r) & \geqslant\left(\tilde{g}^{\prime \prime}(r)-\tilde{g}^{\prime}(r) \eta_{w}(r)\right) g(r)^{2}+m \tilde{g}^{\prime}(r)\left(\eta_{w}(r)-h(r)\right) \\
& =\lim _{k \rightarrow \infty} \frac{-k f_{k-1}(r)}{f_{k}(0)}=\lim _{k \rightarrow \infty} \frac{-k f_{k-1}(0)}{f_{k}(0)} \frac{f_{k-1}(r)}{f_{k-1}(0)} \\
& =-\lambda_{1}\left(B_{s(R)}^{W}\right) \tilde{g} \circ r=-\lambda_{1}\left(B_{s(R)}^{W}\right) v .
\end{aligned}
$$

Therefore,

$$
\sup _{D_{R}}\left\{-\Delta^{P} v / v\right\} \leqslant \lambda_{1}\left(B_{s(R)}^{W}\right)
$$

Since $v_{\mid \partial D_{r}}=0$, Barta's inequalities give us the result.

Theorem 4.2. Let $\left\{N^{n}, P^{m}, C_{w, 1, h}^{m}\right\}$ denote a comparison constellation bounded from above. Assume that $M_{W}^{m}=C_{w, 1, h}^{m}$ is w-balanced from below. Let $D_{R}$ be a smooth precompact extrinsic $R$-ball in $P^{m}$, with center at a point $p \in P$ which also serves as a pole in $N$. Then we have

$$
\lambda_{1}\left(D_{R}\right) \geqslant \lambda_{1}\left(B_{R}^{W}\right)
$$

Suppose moreover that

$$
(m-1) \cdot \inf _{r \in[0, R]} \eta_{w}(r) \geqslant m \cdot \sup _{r \in[0, R]} h(r)
$$

Then

$$
\lambda_{1}\left(B_{R}^{W}\right) \geqslant \frac{1}{4}\left((m-1) \cdot\left(\inf _{r \in[0, R]} \eta_{w}(r)\right)-m \cdot\left(\sup _{r \in[0, R]} h(r)\right)\right)^{2},
$$

where $B_{R}^{W}(\widetilde{p})$ is the pole centered geodesic ball in the model space $M_{W}^{m}$. 
Proof. The proof follows the line of the proof of Theorem 4.1. Let $g_{\infty}(r)$ be the eigenfunction of $\lambda_{1}\left(B_{R}^{W}\right)$ obtained in Theorem 3.6 and given by

$$
g_{\infty}(r)=\lim _{k \rightarrow \infty} \frac{\tilde{u}_{k}^{W}(r)}{\tilde{u}_{k}^{W}(0)}
$$

In this case the stretching function is the identity, so we transplant $g_{\infty}$ to $D_{R}$ composing it with the smooth distance to the pole $p$ on $N$. We obtain the radial function $v: D_{R} \rightarrow \mathbb{R}$ given by $v(q)=g_{\infty}(r(q))$. As before, it is easy to check that

$$
\begin{aligned}
\Delta^{P} v=\Delta^{P}\left(g_{\infty} \circ r\right) & \leqslant g_{\infty}^{\prime \prime}(r)-g_{\infty}^{\prime}(r) \eta_{w}(r)+m g_{\infty}^{\prime}(r)\left(\eta_{w}(r)-h(r)\right) \\
& =-\lambda_{1}\left(B_{R}^{W}\right) g_{\infty} \circ r=-\lambda_{1}\left(B_{R}^{W}\right) v .
\end{aligned}
$$

Therefore,

$$
\inf _{D_{R}}\left\{-\Delta^{P} v / v\right\} \geqslant \lambda_{1}\left(B_{R}^{W}\right),
$$

and since $v_{\mid \partial D_{r}}=0$, Barta's inequalities again give us the first inequality.

To prove inequality (4.4), we follow the argument in Theorem 2 in [CL] - see also the proof of Lemma 2.3 in [BM] - and use the variational Rayleigh formulation of the first Dirichlet eigenvalue of the pole-centered geodesic ball $B_{R}^{W}(\widetilde{p})$. Given a smooth function $f \in C_{0}^{\infty}\left(B_{R}^{W}(\widetilde{p})\right)$, let us consider the vector field $X=\nabla^{M_{W}^{m}} \mu(r)$, where $\mu(r)$ is any smooth radial function. Following the lines of [CL] and computing $\operatorname{div}\left(f^{2} X\right)$, we then have for any $\varepsilon>0$ (a parameter that we shall fix later):

$$
\int_{B_{R}^{W}}\left|\mu^{\prime}(r)\right|\left\|\nabla^{M_{W}^{m}} f\right\|^{2} d \sigma \geqslant \int_{B_{R}^{W}} f^{2}\left(\varepsilon \Delta^{M_{W}^{m}} \mu(r)-\varepsilon^{2}\left|\mu^{\prime}(r)\right|\right) d \sigma
$$

We consider $\mu(r)=r$. The vector field $X=\nabla^{M_{W}^{m}} \mu(r)$ is not necessarily smooth, but we may then approximate it by the smooth field $X=\nabla^{M_{W}^{m} r^{\alpha}}, 1<\alpha<2$, in the punctured ball $B_{R}^{W}(\widetilde{p})-B_{\delta}^{W}(\widetilde{p})$ and recover the same conclusion as below following the argument in [BM, p. 286-288] by letting $\alpha \rightarrow 1$ and $\delta \rightarrow 0$. It is straightforward to see, using the definition of isoperimetric comparison space, Definition 2.7, that

$$
\begin{aligned}
\Delta^{M_{W r}^{m}} & =(m-1) \eta_{W}^{\prime}(r)=(m-1) \eta_{w}(r)-m h(r) \\
& \geqslant(m-1) \inf _{r \in[0, R]} \eta_{w}(r)-m \sup _{r \in[0, R]} h(r) .
\end{aligned}
$$

Using this inequality in equation (4.5) with $\mu(r)=r$, we obtain the following inequality, where $L_{R}:=(m-1) \inf _{r \in[0, R]} \eta_{w}(r)-m \sup _{r \in[0, R]} h(r)$ and $Q(\varepsilon):=\varepsilon L_{R}-\varepsilon^{2}$ :

$$
\int_{B_{R}^{W}}\left\|\nabla^{M_{W}^{m}} f\right\|^{2} d \sigma \geqslant \int_{B_{R}^{W}} f^{2}\left(\varepsilon L_{R}-\varepsilon^{2}\right) d \sigma
$$

Since $Q(\varepsilon)$ attains its maximum at $\varepsilon_{0}=\frac{L_{R}}{2}$, with $Q\left(\varepsilon_{0}\right)=\frac{L_{R}^{2}}{4}$, we finally obtain the desired Poincaré inequality for all $f \in C_{0}^{\infty}\left(B_{R}^{W}(\widetilde{p})\right)$ :

$$
\int_{B_{R}^{W}}\left\|\nabla^{M_{W}^{m}} f\right\|^{2} d \sigma \geqslant \frac{L_{R}^{2}}{4} \int_{B_{R}^{W}} f^{2} d \sigma,
$$


which gives the Rayleigh quotient inequality for the first eigenvalue of $B_{R}^{W}(\widetilde{p})$ :

$$
\lambda_{1}\left(B_{R}^{W}\right) \geqslant \frac{L_{R}^{2}}{4}
$$

4.2. Extrinsic comparison. Second strategy. We present an alternative proof of the first inequality in (4.1) from Theorem 4.1 using Theorem 1.1 and previous results from [HMP2]:

Theorem 4.3 (Second proof of the first inequality of (4.1)). Let $\left\{N^{n}, P^{m}, C_{w, g, h}^{m}\right\}$ denote a comparison constellation bounded from below in the sense of Definition 2.11. Assume that $M_{W}^{m}=C_{w, g, h}^{m}$ is w-balanced from below. Let $D_{R}$ be a smoothly precompact extrinsic $R$-ball in $P^{m}$, with center at a point $p \in P$ which also serves as a pole in $N$.

Then,

$$
\lambda_{1}\left(D_{R}\right) \leqslant \lambda_{1}\left(B_{s(R)}^{W}\right)
$$

where $B_{s(R)}^{W}$ is the geodesic ball in $M_{W}^{m}$.

Proof. We here show how to obtain inequality $\lambda_{1}\left(D_{R}\right) \leqslant \lambda_{1}\left(B_{s(R)}^{W}\right)$ using the description of the first Dirichlet eigenvalue of a smooth precompact domain $D$ in a Riemannian manifold given by P. McDonald and R. Meyers in [McMe]. When $D=D_{R}$, we have

$$
\lambda_{1}\left(D_{R}\right)=\sup \left\{\eta \geqslant 0: \lim _{n \rightarrow \infty} \sup \left(\frac{\eta}{2}\right)^{n} \frac{\mathcal{A}_{n}\left(D_{R}\right)}{\Gamma(n+1)}<\infty\right\} .
$$

On the other hand, we have, under the assumptions of the theorem, the following inequalities concerning the mean exit time moment spectrum of the extrinsic balls, see [HMP2]:

$$
\frac{\mathcal{A}_{n}\left(D_{R}\right)}{\operatorname{Vol}\left(\partial D_{R}\right)} \geqslant \frac{\mathcal{A}_{n}\left(B_{s(R)}^{W}\right)}{\operatorname{Vol}\left(\partial B_{s(R)}^{W}\right)} \quad \text { for all } n \in \mathbb{N}
$$

And moreover, the following inequalities concerning its volume, see [HMP1]:

$$
\begin{aligned}
& \frac{\operatorname{Vol}\left(\partial D_{R}\right)}{\operatorname{Vol}\left(D_{R}\right)} \leqslant \frac{\operatorname{Vol}\left(\partial B_{s(R)}^{W}\right)}{\operatorname{Vol}\left(B_{s(R)}^{W}\right)}, \\
& \operatorname{Vol}\left(D_{R}\right) \leqslant \operatorname{Vol}\left(B_{s(R)}^{W}\right) .
\end{aligned}
$$

Then, using inequality (4.12) the set

$$
H_{1}:=\left\{\eta \geqslant 0: \lim _{n \rightarrow \infty} \sup \left(\frac{\eta}{2}\right)^{n} \frac{\mathcal{A}_{n}\left(D_{R}\right)}{\Gamma(n+1)}<\infty\right\}
$$

is included in the set

$$
H_{2}:=\left\{\eta \geqslant 0: \lim _{n \rightarrow \infty} \sup \left(\frac{\eta}{2}\right)^{n} \frac{\mathcal{A}_{n}\left(B_{s(R)}^{W}\right)}{\Gamma(n+1)} \frac{\operatorname{Vol}\left(\partial D_{R}\right)}{\operatorname{Vol}\left(\partial B_{s(R)}^{W}\right)}<\infty\right\},
$$


so we have

$$
\begin{aligned}
& \lambda_{1}\left(D_{R}\right)=\sup \left\{\eta \geqslant 0: \lim _{n \rightarrow \infty} \sup \left(\frac{\eta}{2}\right)^{n} \frac{\mathcal{A}_{n}\left(D_{R}\right)}{\Gamma(n+1)}<\infty\right\} \\
& \quad \leqslant \sup \left\{\eta \geqslant 0: \lim _{n \rightarrow \infty} \sup \left(\frac{\eta}{2}\right)^{n} \frac{\mathcal{A}_{n}\left(B_{s(R)}^{W}\right)}{\Gamma(n+1)} \frac{\operatorname{Vol}\left(\partial D_{R}\right)}{\operatorname{Vol}\left(\partial B_{s(R)}^{W}\right)}<\infty\right\} \\
& =\frac{\operatorname{Vol}\left(\partial D_{R}\right)}{\operatorname{Vol}\left(\partial B_{s(R)}^{W}\right)} \lambda_{1}\left(B_{s(R)}^{W}\right) .
\end{aligned}
$$

Inequalities (4.13) give the desired inequality.

In the same vein we also present the corresponding alternative proof of the eigenvalue comparison inequality (4.2) from Theorem 4.2 again using Theorem 1.1 and [HMP2]:

Theorem 4.4 (Second proof of inequality (4.2)). Let $\left\{N^{n}, P^{m}, C_{w, 1, h}^{m}\right\}$ denote a comparison constellation bounded from above. Assume that $M_{W}^{m}=C_{w, 1, h}^{m}$ is w-balanced from below. Let $D_{R}$ be a smooth precompact extrinsic $R$-ball in $P^{m}$, with center at a point $p \in P$ which also serves as a pole in $N$. Then we have

$$
\lambda_{1}\left(D_{R}\right) \geqslant \lambda_{1}\left(B_{R}^{W}\right),
$$

where $B_{R}^{W}(\widetilde{p})$ is the pole centered geodesic ball in the model space $M_{W}^{m}$.

If $M_{W}^{m}$ is strictly balanced (in the sense that inequality (2.10) is an strict inequality for all radius r) equality in (4.15) for some fixed radius $R_{0}$ implies that $D_{R_{0}}$ is a geodesic cone in $N$. If $P$ is minimal and $N=\mathbb{H}^{n}(b)$ is the hyperbolic space, we then have that $P$ is a totally geodesic submanifold in $\mathbb{H}^{n}(b)$.

Proof. As in the above theorem, we can again obtain inequality $\lambda_{1}\left(D_{R}\right) \geqslant \lambda_{1}\left(B_{R}^{W}\right)$ using the description (4.11) of the first Dirichlet eigenvalue given in [McMe] restricted to the extrinsic balls. Under the assumptions of the theorem we now have the following inequalities concerning the mean exit time moment spectrum of the extrinsic balls, see [HMP2]:

$$
\frac{\mathcal{A}_{n}\left(D_{R}\right)}{\operatorname{Vol}\left(\partial D_{R}\right)} \leqslant \frac{\mathcal{A}_{n}\left(B_{R}^{W}\right)}{\operatorname{Vol}\left(\partial B_{R}^{W}\right)} \text { for all } n \in \mathbb{N}
$$

And the corresponding inequalities concerning the relative volumes, see [HMP1]:

$$
\begin{aligned}
& \frac{\operatorname{Vol}\left(\partial D_{R}\right)}{\operatorname{Vol}\left(D_{R}\right)} \geqslant \frac{\operatorname{Vol}\left(\partial B_{R}^{W}\right)}{\operatorname{Vol}\left(B_{R}^{W}\right)}, \\
& \operatorname{Vol}\left(D_{R}\right) \geqslant \operatorname{Vol}\left(B_{R}^{W}\right) .
\end{aligned}
$$

Then, using inequality (4.16) the set

$$
H_{2}:=\left\{\eta \geqslant 0: \lim _{n \rightarrow \infty} \sup \left(\frac{\eta}{2}\right)^{n} \frac{\mathcal{A}_{n}\left(B_{R}^{W}\right)}{\Gamma(n+1)} \frac{\operatorname{Vol}\left(\partial D_{R}\right)}{\operatorname{Vol}\left(\partial B_{R}^{W}\right)}<\infty\right\}
$$

is included in the set

$$
H_{1}:=\left\{\eta \geqslant 0: \lim _{n \rightarrow \infty} \sup \left(\frac{\eta}{2}\right)^{n} \frac{\mathcal{A}_{n}\left(D_{R}\right)}{\Gamma(n+1)}<\infty\right\},
$$

so we have 


$$
\begin{aligned}
\lambda_{1}\left(D_{R}\right) & =\sup \left\{\eta \geqslant 0: \lim _{n \rightarrow \infty} \sup \left(\frac{\eta}{2}\right)^{n} \frac{\mathcal{A}_{n}\left(D_{R}\right)}{\Gamma(n+1)}<\infty\right\} \\
& \geqslant \sup \left\{\eta \geqslant 0: \lim _{n \rightarrow \infty} \sup \left(\frac{\eta}{2}\right)^{n} \frac{\mathcal{A}_{n}\left(B_{s(R)}^{W}\right)}{\Gamma(n+1)} \frac{\operatorname{Vol}\left(\partial D_{R}\right)}{\operatorname{Vol}\left(\partial B_{R}^{W}\right)}<\infty\right\} \\
= & \frac{\operatorname{Vol}\left(\partial D_{R}\right)}{\operatorname{Vol}\left(\partial B_{R}^{W}\right)} \lambda_{1}\left(B_{R}^{W}\right) .
\end{aligned}
$$

Then we have inequality $\lambda_{1}\left(D_{R}\right) \geqslant \lambda_{1}\left(B_{R}^{W}\right)$ using now inequalities (4.17).

This alternative proof allows us to discuss the equality case as in the paper [HMP2]: If $M_{W}^{m}$ is strictly balanced, (in the sense that inequality (2.10) is an strict inequality for all radius $r)$, equality $\lambda_{1}\left(D_{R_{0}}\right)=\lambda_{1}\left(B_{R_{0}}^{W}\right)$ for some fixed radius $R_{0}$ implies the equality between the volumes $\operatorname{Vol}\left(D_{R_{0}}\right)=\operatorname{Vol}\left(B_{R_{0}}^{W}\right)$, which implies in itself that $\nabla^{P} r=\nabla^{N_{r}}$ and $D_{R_{0}}$ is a geodesic cone in $N$ swept out by the radial geodesics from the center $p$ of the ball. If $P$ is minimal and $N=\mathbb{H}^{n}(b)$ is the hyperbolic space, we then have that $P$ is a totally geodesic submanifold in $\mathbb{H}^{n}(b)$.

4.3. Intrinsic comparison. We finally consider the intrinsic consequences of Theorems 4.1 and 4.2 assuming that $P^{m}=N^{n}$. In this case, the extrinsic distance to the pole $p$ becomes the intrinsic distance in $N$, so, for all $r$ the extrinsic domains $D_{r}$ become the geodesic balls $B_{r}^{N}$ of the ambient manifold $N$. Then, for all $x \in P$

$$
\begin{aligned}
\nabla^{P} r(x) & =\nabla r(x), \\
H_{P}(x) & =0 .
\end{aligned}
$$

As a consequence, $\left\|\nabla^{P} r\right\|=1$, so $g(r(x))=1$ and $\mathcal{C}(x)=h(r(x))=0$, the stretching function becomes the identity $s(r)=r, W(s(r))=w(r)$, and the isoperimetric comparison space $C_{w, g, h}^{m}$ is reduced to the auxiliary model space $M_{w}^{m}$.

As a corollary of the proofs of the theorems above we therefore have:

Theorem 4.5. Let $B_{R}^{N}$ be a geodesic ball of a complete Riemannian manifold $N^{n}$ with a pole $p$ and suppose that the $p$-radial sectional curvatures of $N^{n}$ are bounded from below by the $p_{w}$-radial sectional curvatures of a $w$-model space $M_{w}^{n}$. Then

$$
\lambda_{1}\left(B_{R}^{N}\right) \leqslant \lambda_{1}\left(B_{R}^{w}\right)
$$

where $B_{R}^{w}$ is the $p_{w}$-centered geodesic ball in $M_{w}^{n}$. If $M_{w}^{m}$ is strictly balanced, then equality in (4.19) for some fixed radius $R_{0}$ implies that $B_{R_{0}}^{N}$ and $B_{R_{0}}^{w}$ are isometric.

Theorem 4.6. Let $B_{R}^{N}$ be a geodesic ball of a complete Riemannian manifold $N^{n}$ with a pole $p$ and suppose that the $p$-radial sectional curvatures of $N^{n}$ are bounded from above by the $p_{w}$-radial sectional curvatures of a w-model space $M_{w}^{n}$. Then

$$
\lambda_{1}\left(B_{R}^{N}\right) \geqslant \lambda_{1}\left(B_{R}^{w}\right)
$$

where $B_{R}^{w}$ is the geodesic ball in $M_{w}^{n}$. If $M_{w}^{m}$ is strictly balanced, then equality in (4.20) for some fixed radius $R_{0}$ implies that $B_{R_{0}}^{N}$ and $B_{R_{0}}^{w}$ are isometric.

Remark 4.7. The equality statements - now in both theorems - come from the fact that equality for some fixed radius $R_{0}$ in inequalities (4.19) and (4.20), leads via arguments in Theorems 4.3 and 4.4 to the equality among the volumes of the geodesic balls $B_{R_{0}}^{N}$ and $B_{R_{0}}^{w}$. 
We conclude that they are therefore isometric using a direct application of Bishop's volume comparison theorem, as found e.g. in Corollary 3.2, Chapter IV in [S].

\section{REFERENCES}

[Ba] Catherine Bandle. Isoperimetric inequalities and applications, volume 7 of Monographs and Studies in Mathematics. Pitman (Advanced Publishing Program), Boston, Mass., 1980.

[BBC] Rodrigo Bañuelos, M. van den Berg, and Tom Carroll. Torsional rigidity and expected lifetime of Brownian motion. J. London Math. Soc. (2), 66(2):499-512, 2002.

[BaBe1] Cleon S. Barroso and G. Pacelli Bessa. A note on the first eigenvalue of spherically symmetric manifolds. Mat. Contemp., 30:63-69, 2006. XIV School on Differential Geometry (Portuguese).

[BaBe2] Cleon S. Barroso and G. Pacelli Bessa. Lower bounds for the first Laplacian eigenvalue of geodesic balls of spherically symmetric manifolds. Int. J. Appl. Math. Stat., 6(D06):82-86, 2006.

[Bar] J Barta. Sur le vibration fondamentale d'une membrane. C. R. Acad. Sci., 204:472-473, 1937.

[BM] G. Pacelli Bessa and J. Fábio Montenegro. Eigenvalue estimates for submanifolds with locally bounded mean curvature. Ann. Global Anal. Geom., 24(3):279-290, 2003.

[BCG] C. Betz, G. A. Cámera, and H. Gzyl. Bounds for the first eigenvalue of a spherical cap. Appl. Math. Optim., 10(3):193-202, 1983.

[Ch1] Isaac Chavel. Eigenvalues in Riemannian geometry, volume 115 of Pure and Applied Mathematics. Academic Press Inc., Orlando, FL, 1984. Including a chapter by Burton Randol, With an appendix by Jozef Dodziuk.

[Ch2] Isaac Chavel. Isoperimetric inequalities, volume 145 of Cambridge Tracts in Mathematics. Cambridge University Press, Cambridge, 2001. Differential geometric and analytic perspectives.

[Chg1] Shiu Yuen Cheng. Eigenfunctions and eigenvalues of Laplacian. In Differential geometry (Proc. Sympos. Pure Math., Vol. XXVII, Stanford Univ., Stanford, Calif., 1973), Part 2, pages 185-193. Amer. Math. Soc., Providence, R.I., 1975.

[Chg2] Shiu Yuen Cheng. Eigenvalue comparison theorems and its geometric applications. Math. Z., 143(3):289-297, 1975.

[CL] Leung-Fu Cheung and Pui-Fai Leung. Eigenvalue estimates for submanifolds with bounded mean curvature in the hyperbolic space. Math. Z., 236(3):525-530, 2001.

[DCW] M. P. do Carmo and F. W. Warner. Rigidity and convexity of hypersurfaces in spheres. J. Differential Geometry, 4:133-144, 1970.

[Dy] E. B. Dynkin. Markov processes. Vols. I, II, volume 122 of Translated with the authorization and assistance of the author by J. Fabius, V. Greenberg, A. Maitra, G. Majone. Die Grundlehren der Mathematischen Wissenschaften, Bände 121. Academic Press Inc., Publishers, New York, 1965.

[FH] S. Friedland and W. K. Hayman. Eigenvalue inequalities for the Dirichlet problem on spheres and the growth of subharmonic functions. Comment. Math. Helv., 51(2):133-161, 1976.

[G] Michael E. Gage. Upper bounds for the first eigenvalue of the Laplace-Beltrami operator. Indiana Univ Math. J., 29(6):897-912, 1980.

[GreW] R. E. Greene and H. Wu. Function theory on manifolds which possess a pole, volume 699 of Lecture Notes in Mathematics. Springer, Berlin, 1979.

[Gri] Alexander Grigor'yan. Analytic and geometric background of recurrence and non-explosion of the Brownian motion on Riemannian manifolds. Bull. Amer. Math. Soc. (N.S.), 36(2):135-249, 1999.

[HMP1] A. Hurtado, S. Markvorsen, and V. Palmer. Torsional rigidity of submanifolds with controlled geometry. Math. Ann., 344(3):511-542, 2009.

[HMP2] A. Hurtado, S. Markvorsen, and V. Palmer. Comparison of exit moment spectra for extrinsic metric balls. Potential Anal., 36(1):137-153, 2012.

[JK] L. Jorge and D. Koutroufiotis. An estimate for the curvature of bounded submanifolds. Amer. J. Math., 103(4):711-725, 1981

[M] Steen Markvorsen. On the mean exit time from a minimal submanifold. J. Differential Geom., 29(1):1-8, 1989.

[MM] Steen Markvorsen and Maung Min-Oo. Global Riemannian geometry: curvature and topology. Advanced Courses in Mathematics. CRM Barcelona. Birkhäuser Verlag, Basel, 2003.

[MP1] Steen Markvorsen and Vicente Palmer. How to obtain transience from bounded radial mean curvature. Trans. Amer. Math. Soc., 357(9):3459-3479 (electronic), 2005.

[MP2] Steen Markvorsen and Vicente Palmer. Torsional rigidity of minimal submanifolds. Proc. London Math. Soc. (3), 93(1):253-272, 2006.

[MP3] Steen Markvorsen and Vicente Palmer. Extrinsic isoperimetric analysis of submanifolds with curvatures bounded from below. J. Geom. Anal., 20(2):388-421, 2010.

[MaT] Tadayuki Matsuzawa and Shûkichi Tanno. Estimates of the first eigenvalue of a big cup domain of a 2-sphere. Compositio Math., 47(1):95-100, 1982. 
[Mc1] Patrick McDonald. Isoperimetric conditions, Poisson problems, and diffusions in Riemannian manifolds. Potential Anal., 16(2):115-138, 2002.

[Mc2] Patrick McDonald. Exit times, moment problems and comparison theorems. Potential Anal., 38:13651372, 2013.

[McMe] Patrick McDonald and Robert Meyers. Dirichlet spectrum and heat content. J. Funct. Anal., 200(1):150159, 2003.

[McK] H. P. McKean. An upper bound to the spectrum of $\Delta$ on a manifold of negative curvature. J. Differential Geometry, 4:359-366, 1970.

[O'N] Barrett $\mathrm{O}^{\prime}$ Neill. Semi-Riemannian geometry, volume 103 of Pure and Applied Mathematics. Academic Press Inc. [Harcourt Brace Jovanovich Publishers], New York, 1983. With applications to relativity.

[Pa] Vicente Palmer. Mean exit time from convex hypersurfaces. Proc. Amer. Math. Soc., 126(7):2089-2094, 1998.

[Pe] Peter Petersen. Riemannian geometry, volume 171 of Graduate Texts in Mathematics. Springer, New York, second edition, 2006.

[Pi] Mark A. Pinsky. The first eigenvalue of a spherical cap. Appl. Math. Optim., 7(2):137-139, 1981.

[PS] G. Pólya and G. Szegö. Isoperimetric Inequalities in Mathematical Physics. Annals of Mathematics Studies, no. 27. Princeton University Press, Princeton, N. J., 1951.

[Po] George Pólya. Torsional rigidity, principal frequency, electrostatic capacity and symmetrization. Quart. Appl. Math., 6:267-277, 1948.

[S] Takashi Sakai. Riemannian geometry, volume 149 of Translations of Mathematical Monographs. American Mathematical Society, Providence, RI, 1996. Translated from the 1992 Japanese original by the author.

[Sa] Sadao Sato. Barta's inequalities and the first eigenvalue of a cap domain of a 2-sphere. Math. Z., 181(3):313-318, 1982.

[Sav] Alessandro Savo. On the lowest eigenvalue of the Hodge Laplacian on compact, negatively curved domains. Ann. Global Anal. Geom., 35(1):39-62, 2009.

[Sp] Michael Spivak. A comprehensive introduction to differential geometry. Vol. V. Publish or Perish Inc., Wilmington, Del., second edition, 1979.

[VBG] M. van den Berg and P. B. Gilkey. Heat content and a Hardy inequality for complete Riemannian manifolds. Bull. London Math. Soc., 36(5):577-586, 2004.

[Y] Shing Tung Yau. Isoperimetric constants and the first eigenvalue of a compact Riemannian manifold. Ann. Sci. École Norm. Sup. (4), 8(4):487-507, 1975.

Departamento de Geometría y Topología, Universidad de Granada, E-18071, Spain.

E-mail address: ahurtado@ugr.es

DTU Compute, Mathematics, DK-2800 KGs. Lyngby, DenMark

E-mail address: stema@dtu.dk

Departament de Matemàtiques-INIT, Universitat Jaume I, Castelló, Spain.

E-mail address: palmer@mat.uji.es 